\title{
UN ANÁLISIS VAR ESTRUCTURAL DE POLÍTICA MONETARIA EN COLOMBIA*
}

\author{
ALEJANDRO RAMÍREZ VIGOYA** \& HERNANDO RODRÍGUEZ ZAMBRANO***** \\ UNIVERSIDAD MILITAR NUEVA GRANADA
}

Recibido/ Received/ Recebido: 29/05/2013 - Aceptado/ Accepted / Aprovado: 07/10/2013

\begin{abstract}
Resumen
Este trabajo utiliza la metodología VAR estructural, con restricciones de corto plazo impuestas por el modelo macroeconómico de comportamiento AD-AS, para identificar las relaciones contemporáneas entre las variables endógenas del sistema que imponen las curvas oferta agregada, la LM y la MP. También se analizan los efectos dinámicos de choques en la tasa de intervención del Banco de la República sobre el crecimiento económico y la inflación. Los resultados de las curvas contemporáneas MP indican que hay una relación estadísticamente significativa y positiva contemporánea entre las variables tasa de intervención del Banco de la República y la inflación. El análisis dinámico vía respuesta impulso indica que solamente los choques positivos de las diferencias de la tasa de intervención tienen efectos negativos rezagados y cíclicos sobre la inflación. Choques positivos de política monetaria tienen efectos negativos y significativos sobre el crecimiento económico. A través de la descomposición de varianza se encontró que ante choques de política monetaria se presentan mayores efectos acumulados en el crecimiento económico que en la inflación, y en esta última solamente cuando la tasa de intervención está en diferencias.

Palabras clave: Vectores autorregresivos estructurales, Política monetaria, Crecimiento económico.
\end{abstract}

\section{STRUCTURAL VAR ANALYSIS OF MONETARY POLICY IN COLOMBIA}

\begin{abstract}
This article is based on structural VAR methodology, considering short term restrictions imposed by an AD-AS macroeconomic model to identify contemporary relations among system endogenous variables imposed aggregated supply, LM and MP curves. Dynamic shock effects in the Central Bank's (Banco de la República) intervention rate are also analyzed on economic growth and inflation. Results of MP Contemporary curves point out that the relationship is statistically significant and positive contemporary among Central Bank's (Banco de la República) intervention rate and inflation. The dynamic path through impulse response analysis indicates that only positive shocks of intervention
\end{abstract}

* Este trabajo es el resultado del proyecto de investigación: "Un Análisis Estructural de Política Monetaria en Colombia", de la línea de Políticas Públicas del grupo de investigación CIE.

*** Profesor de macroeconomía de la Universidad Militar Nueva Granada. Correo electrónico: alejandro.ramirez@unimilitar.edu.co, alejram@uniandes.edu.co.

**** Profesor de planta de la Universidad Militar Nueva Granada. Economista y magister en economía de la Universidad de los Andes. Correo electrónico: hernando.rodriguez@unimilitar.edu.co 
rate in difference have lagged and cyclic negative effects on inflation. Positive shocks to monetary policy have negative and significant effects on economic growth. Through variance decomposition, the author found that monetary policy shocks present higher accumulated effects in economic growth than in inflation and in the case of inflation these effects only present when intervention rate is a difference ecuation.

Key words: Quality Management, Production process standardization, Process management.

\title{
UMA ANÁLISE VAR ESTRUTURAL DE POLÍTICA MONETÁRIA NA COLÔMBIA
}

\begin{abstract}
Resumo
Este trabalho utiliza a metodologia VAR estrutural, com restrições de curto prazo impostas pelo modelo macroeconômico de comportamento AD-AS, para identificar as relações contemporâneas entre as variáveis endógenas do sistema, que impõem as curvas de oferta agregada, a LM e a MP. Também se analisam os efeitos dinâmicos de choques na taxa de intervenção do Banco da República sobre o crescimento econômico e sobre a inflação. Os resultados das curvas contemporâneas MP indicam que há uma relação estatisticamente significativa e positiva contemporânea entre as variáveis taxa de intervenção do Banco da República e a inflação. A análise dinâmica via resposta impulso indica que somente os choques positivos das diferenças da taxa de intervenção têm efeitos negativos retardados e cíclicos sobre a inflação. Choques positivos de política monetária têm efeitos negativos e significativos sobre o crescimento econômico. Através da decomposição de variância se encontrou que ante choques de política monetária se apresentam maiores efeitos acumulados no crescimento econômico do que na inflação, e nesta última somente quando a taxa de intervenção está em diferenças.
\end{abstract}

Palavras chave: Vectores autorregressivos estruturais, Política monetária, Crescimento econômico.

Ramírez, A. \& Rodríguez, H. (2013) Un análisis VAR estructuralde política monetaria en Colombia. En: Revista de la Facultad de Ciencias Económicas de la Universidad Militar Nueva Granada. rev. fac.cienc.econ., XXI (2)

JEL: E52.

\section{Introducción}

La metodología VAR estructuralse cuenta dentro de las herramientas econométricas aplicables a ecuaciones simultáneas, que parten de modelos macroeconómicos de comportamiento. Muchos trabajos suponen ciertas restricciones contemporáneas entre variables macroeconómicas, pero no es clara la relación implícita entre éstas.

En Colombia hay trabajos interesantes que utilizan la metodología VAR estructural como el de Misas
\& López (1998), en el cual analizan modelos de aumento de la inflación y calculan el producto potencial para Colombia. También está el trabajo de Echavarría et al. (2009), en el cual encuentran el impacto de un doble choque de política monetaria convencional y de intervenciones cambiarias sobre la tasa de interés, la tasa de cambio y otras variables en el sistema.

Por otro lado están los trabajos que utilizan diferentes metodologías como el de González et al. (2011) donde analizan las relaciones entre el crecimiento del 
producto y el empleo, utilizando modelos SVAR y de equilibrio general. En este mismo tema está el trabajo de Campos (2006) que analiza la brecha entre el PIB potencial y el observado utilizando la metodología SVAR.

En cuanto a efectos coyunturales de decisiones de política monetaria está el trabajo de Sarmiento \& Ramírez (2005), en el cual calculan los efectos del proceso des-inflacionario en Colombia a partir de choques negativos sobre la cantidad de dinero y sus efectos sobre la tasa de interés, la tasa de cambio, la producción y los precios.

Aunque la metodología VAR estructurales conocida y utilizada desde diferentes tópicos en Colombia, la intención de este trabajo es explicar de una manera pormenorizada los procedimientos matemáticos y econométricos tanto del modelo macroeconómico de comportamiento (AD-AS), que es una forma de especificación del modelo IS-LM, como del proceso econométrico que utiliza y desarrolla la metodología VAR estructural. La especificación macroeconómica y econométrica es utilizada por Mihira \& Sugihara (2000), entre otras cuatro especificaciones más para calcular modelos VAR estructural bajo diferentes restricciones. En este trabajo nos concentramos en una de estas especificaciones macroeconómicas, el modelo AD-AS, que al aplicarlo para Colombia nos dará herramientas estadísticas para entender los efectos de choques de política monetaria tanto en el corto como en el largo plazo. Para el análisis de corto plazo se utiliza la metodología conocida como descomposición de Choleski, para entender las relaciones contemporáneas entre las variables del sistema. En el largo plazo para calcular la contabilidad de las innovaciones, es decir, los efectos dinámicos, se utiliza respuesta impulso y descomposición de varianza para medir los efectos rezagados de choques de política monetaria.

El trabajo aquí desarrollado intenta pormenorizar los pasos a seguir en el planteamiento del modelo macroeconómico y las implicaciones que tiene en los resultados del VAR estructural tanto en las relaciones contemporáneas como en los efectos de largo plazo. Se utiliza un modelo VAR estructural del tipo K, es decir, con relaciones contemporáneas entre las variables endógenas del sistema.

La primera parte del trabajo es esta introducción. En la segunda parte se plantea un modelo AD-AS macroeconómico de comportamiento, en el cual se pueden separar los choques de AS (Oferta agregada), de MP (Política monetaria) y de LM (Mercado monetario).

En esta parte lo que interesa realmente es analizar las relaciones contemporáneas implícitas en la curva MP, es decir, entre la tasa de intervención del Banco de la República con la inflación y el crecimiento económico y, sobre todo, la significancia de los coeficientes entre las variables en el hiperplano MP. También es importante encontrar los efectos de largo plazo de choques de la variable utilizada como herramienta de política monetaria, la tasa de intervención del Banco de la República, sobre el crecimiento del pib y sobre la variación de los precios.

Cabe anotar que en el modelo utilizado no se analizan los mecanismos de transmisión de política monetaria vía tasa de intervención. El modelo utilizado AD-AS es de los más sencillos, pero es importante entender el planteamiento del modelo y los efectos directos, ya sea en forma contemporánea o en el largo plazo.

En la tercera parte nos basamos en Amissano \& Giannini (1997), para resolver la matemática del VAR estándar y del VAR estructural y sobre todo interesa que quede clara la relación entre los errores de cada uno de los modelos, que es fundamental para entender el funcionamiento del VAR estructural. La cuarta parte es un análisis de la especificación de los datos, que es importante para explicar las relaciones contemporáneas entre las variables del sistema y los efectos de largo plazo de las políticas monetarias. En la quinta parte se especifican los diferentes modelos a correr, según las series utilizadas. En la sexta parte, que es la más importante, se presentan los resultados de las relaciones contemporáneas y de largo plazo entre las variables que explican la política monetaria. La última parte del trabajo son las conclusiones del mismo. 


\section{Modelo Macroeconómico Estructural}

Se parte de uno de los modelos macroeconómicos de comportamiento AD-AS (oferta agregada - demanda agregada) utilizado por Mihira y Sugihara (2000), que tiene la siguiente estructura implícita de comportamiento:

(1) $y=c(\stackrel{+}{y})+i(\stackrel{+}{y}, \bar{R}+\bar{P} e)+\bar{g}$

IS: Demanda real

(2) $M / P=m^{d}(\stackrel{+}{y}, \bar{R})$

LM: Demanda de dinero

(3) $y=F\left[L^{d}(\bar{W} / P) ; \bar{k}, \bar{A}\right]=y^{s}(\stackrel{+}{P} / \bar{W} ; \bar{k}, \bar{A})$

AS: Oferta agregada

(4) $R=R^{p}\left(y-\bar{y}^{*}, P-\bar{P}^{*}\right)=R^{p}\left(\stackrel{+}{y}, \stackrel{+}{P} / \bar{P}_{-1} ; \bar{y}^{*}, \bar{P}^{*}\right)$

MP: Política monetaria

Las variables endógenas son: producto y, consumo c, inversión i, tasa de interés de política monetaria $\mathrm{R}$, cantidad de dinero $\mathrm{M}$, precios $\mathrm{P} y$ trabajo $\mathrm{L}$.

Las variables exógenas son: inflación Pe, gasto del gobierno g, salarios $\mathrm{W}$, stock de capital $\mathrm{k}$, tecnología A, producción objetivo de política $y^{*}$, e inflación objetivo P*.

La estructura (1) a (4) se puede convertir en un proceso estocástico, para ello se eliminan las variables exógenas y dos de las endógenas, el consumo y el trabajo. En este caso, los cambios sobre las variables endógenas de choques en las variables exógenas se reflejan en los errores.

Reordenando (1) a (4) e incluyendo solamente las variables endógenas producción $\mathrm{Y}$, precios $\mathrm{P}$, tasa de interés de política monetaria $\mathrm{R}$ y cantidad de dinero
$\mathrm{M}$, resulta la siguiente estructura estocástica en forma implícita.

$\left(1^{\prime}\right)$

$$
y=y^{d}(R)+\varepsilon_{I S, Y} \quad \text { IS }
$$

(2')

$$
y=y^{s}(\stackrel{+}{P})+\varepsilon_{A S, Y} \Leftrightarrow P=P^{s}(\stackrel{+}{y})+\varepsilon_{A S, P} \text { AS }
$$

(3’) $R=R^{p}(\stackrel{+}{y}, \stackrel{+}{P})+\varepsilon_{M P, R} \quad \mathrm{MP}$

$$
\text { (4') } M=m^{d}(\stackrel{+}{y}, \stackrel{+}{P}, \bar{R})+\varepsilon_{L M, M} \quad \mathrm{LM}
$$

El anterior modelo implícito se puede convertir en un modelo explícito de comportamiento contemporáneo entre las variables endógenas. Pasando tanto las variables endógenas como los choques (innovaciones) a la izquierda, resulta el siguiente sistema. En el sistema (5) a (8) las variables endógenas son la producción $(\mathrm{Y})$, los precios $(\mathrm{P})$, la tasa de intervención del Banco $(\mathrm{R})$ y la cantidad de dinero (M). Los choques de IS, AS, MP y LM funcionan como variables exógenas, por lo tanto se pueden encontrar los efectos de cambios en los choques sobre las variables endógenas del sistema. La curva MP es una versión simplificada de la "Regla de Taylor".

$$
\begin{array}{cl}
\text { (5) } y+a_{Y R} R-\varepsilon_{I S, Y}=0 & \text { IS } \\
\text { (6) }-a_{P Y} y+P-\varepsilon_{A S, P}=0 & \text { AS } \\
\text { (7) }-a_{R Y} y-a_{R P} P+R-\varepsilon_{M P, R}=0 & \mathrm{MP} \\
\text { (8) }-a_{M Y} y-a_{M P} P+a_{M R} R+M-\varepsilon_{L M, M}=0 & \mathrm{LM}
\end{array}
$$

El sistema (5) a (8) se puede representar en forma de matriz de coeficientes contemporáneos, la cual se utilizará más adelante en el modelo VAR estructuralpara calcular los coeficientes contemporáneos y los choques del modelo estructural. 
(9) $\left[\begin{array}{cccc}1 & 0 & a_{Y R} & 0 \\ -a P Y & 1 & 0 & 0 \\ -a R Y & -a R P & 1 & 0 \\ -a M Y & -a M P & a M R & 1\end{array}\right]\left[\begin{array}{l}Y \\ P \\ R \\ M\end{array}\right]=\left[\begin{array}{l}\varepsilon_{I S, Y} \\ \varepsilon_{A S, P} \\ \varepsilon_{M P, R} \\ \varepsilon_{L M, M}\end{array}\right] \leftrightarrow A_{o} X_{t}=\varepsilon$

La matriz Ao del lado izquierdo en (9) contiene los signos esperados de las relaciones contemporáneas entre las variables endógenas del sistema ${ }^{1}$.

A partir del sistema (5) a (8) se puede hacer análisis de estática comparativa para conocer los efectos de los choques de $\mathrm{IS}(\mathrm{Y}), \mathrm{AS}(\mathrm{P}), \mathrm{MP}(\mathrm{R})$ y $\mathrm{LM}(\mathrm{M})$ sobre cada una de las variables endógenas del sistema. Para analizar los efectos de choques de política monetaria $\mathrm{MP}(\mathrm{R})$ sobre $\mathrm{Y}, \mathrm{P}, \mathrm{R}$ y $\mathrm{M}$, se resuelve por Cramer el sistema ${ }^{2}$ en (10).

$$
\left[\begin{array}{llll}
1 & 0 & a_{\mathrm{YR}} & 0 \\
-\mathrm{aPY} & 1 & 0 & 0 \\
-\mathrm{aRY} & -\mathrm{aRP} & 1 & 0 \\
-\mathrm{aMY} & -\mathrm{aMP} & \mathrm{aMR} & 1
\end{array}\right]\left[\begin{array}{l}
\partial \mathrm{Y} \partial \varepsilon \mathrm{MP} \\
\partial \mathrm{P} \partial \varepsilon \mathrm{MP} \\
\partial \mathrm{R} \partial \mathrm{g} \mathrm{MP} \\
\partial \mathrm{M} \partial \varepsilon \mathrm{MP}
\end{array}\right]=\left[\begin{array}{l}
0 \\
0 \\
1 \\
0
\end{array}\right]
$$

$\mathrm{Al}$ igual que en (10) se resuelven los choques de IS, AS y LM sobre las variables endógenas Y, P, R y M. A continuación se resumen los resultados del análisis de estática comparativa de todos los choques:

$$
\left[\begin{array}{l}
\partial Y \\
\partial P \\
\partial R \\
\partial M
\end{array}\right]=\left[\begin{array}{llll}
+ & - & - & 0 \\
+ & + & - & 0 \\
+ & + & + & 0 \\
? & ? & - & +
\end{array}\right]\left[\begin{array}{c}
\partial I S \\
\partial A S \\
\partial M P \\
\partial L M
\end{array}\right]
$$

Teóricamente los signos esperados de efectos inmediatos, en el modelo macroeconómico de comportamiento AD-AS, de choques de IS, AS, MP y LM sobre el PIB, el IPC, la tasa de interés de política monetaria y la cantidad de dinero son los descritos en (11).

Un choque de IS(Y) aumenta Y, P y R, mientras no se conoce el efecto sobre $\mathrm{M}$.

Un choque de $\mathrm{AS}(\mathrm{P})$ aumenta $\mathrm{P}$ y $\mathrm{R}$ y disminuye $\mathrm{Y}$, no se conoce el efecto sobre $M$.

Un choque de $\mathrm{MP}(\mathrm{R})$ disminuye a $\mathrm{Y}, \mathrm{P}$ y $\mathrm{M}$, mientras aumenta R.

Un choque de $\mathrm{LM}(\mathrm{M})$ no tiene efectos sobre $\mathrm{Y}, \mathrm{P}$ y $\mathrm{R}$, pero si aumenta a $\mathrm{M}$.

\section{Metodología VAR estructural}

La metodología VAR estructural fue popularizada y le dio el premio Nóbel de economía a Christopher Sims en 2011. Sims $(1980,1986,1988)^{3}$ propuso una nueva metodología basada en sistemas de ecuaciones simultáneas en la cual cada variable se regresa en un número finito de rezagos de todas las variables endógenas del sistema. El sistema VAR estructural se fundamenta en el conocimiento de las propiedades estadísticas de las series utilizadas y en las relaciones macroeconómica subyacentes entre las variables.

El conocimiento de las relaciones entre las variables es fundamental para imponer restricciones que se reflejan en los errores del sistema estructural.

Siguiendo a Amissano \& Giannini (1997), existen tres clases de vares estructurales, los modelos $\mathrm{K}$, los modelos $\mathrm{C}$ y los modelos $\mathrm{AB}$.

En los modelo $\mathrm{K}$ se pre-multiplica el sistema var estándar por la matriz de coeficientes contemporáneos, de tal manera que $K A(L) x_{t}=K \varepsilon_{t}$, entonces los errores del VAR estructural son una combinación

\footnotetext{
1 Realmente los signos esperados son los contrarios a los de la matriz Ao en (9), ya que si se despejan Y, P, R y M, entonces los signos de las relaciones contemporáneas son los contrarios.

2 En el anexo 1 se presenta la matemática para el análisis de estática comparativa en (10) para choques de MP sobre Y, P, R y M que se resumen en (11). Los choques de IS, AS y LM se calculan de forma similar a los de MP.

3 Trabajos que le valieron el premio Nóbel de economía 2011, junto a Thomas Sargent.
} 
lineal de los errores del var estándar y de la matriz de coeficientes contemporáneos impuestos por la estructura macroeconómica subyacente. Los efectos dinámicos en estos modelos están afectados por las restricciones contemporáneas impuestas en $\mathrm{K}$.

Un ejemplo de trabajos clásicos de este tipo de modelo es el de Blanchard \& Watson (1986) en el cual analizan los choques que explican las fluctuaciones de los ciclos de negocios, llegando a la conclusión de que hay diferentes choques que explican las fluctuaciones, no solamente, los choques monetarios.

Los modelos $C$ son de la forma $A(L) x_{t}=\varepsilon_{t} y$ $\varepsilon_{t}=C \varepsilon_{t}$, en este caso no hay restricciones en las relaciones contemporáneas. Cada variable en el sistema está afectada por un set de choques ortonormales cuyo impacto está modelado en la matriz C. Como ejemplo de trabajos que utilizan estos modelos están los trabajos de Blanchard \& Quah (1989), y Shapiro \& Watson (1988). En el primero Blanchard \& Quah analizan los efectos de choques tanto de demanda como de oferta en un sistema de dos variables: crecimiento económico y tasa de desempleo, cuando existen restricciones de largo plazo en la matriz $\mathrm{C}$. En el segundo Shapiro \& Watson llegan a la conclusión de que los choques de demanda explican entre el 20 y el 30 por ciento de las fluctuaciones del pib en los ciclos de negocios, Y que los choques tecnológicos explican un 25 por ciento de las fluctuaciones del PIB y una tercera parte de su varianza.

Un trabajo interesante que utiliza tanto modelos $\mathrm{K}$ como C es el de Mihira \& Sugihara (2000), quienes utilizan cinco modelos de comportamiento macroeconómico, incluido uno con bloques recursivos y otro con restricciones de largo plazo a lo Blanchard y Quah. Los autores llegan a la conclusión de que el modelo con bloques recursivos que utiliza la variable IPP (Índice de Precios al Productor) como variable sensitiva es el que mejor explica los efectos de política monetaria.

Los modelos $\mathrm{AB}$ son de la forma: $A A(L) x_{t}=A \varepsilon_{t} \mathrm{y}$ $A \varepsilon_{t}=B \varepsilon_{t}$. En este modelo hay restricciones simultáneas de corto plazo en A y de largo plazo en $\mathrm{B}$.
Trabajos interesantes que utilizan este modelo son los de Bernanke (1986) y Blanchard (1989). Bernanke (1986) se basa en el trabajo ya mencionado de Blanchard \& Quah (1989) y en una metodología alternativa a la que utiliza Sims en los trabajos mencionados anteriormente.

Bernanke parte de la hipótesis de que los choques del crédito no explican nada y que los choques reales lo explican todo. Llega a la conclusión de que ninguna de sus hipótesis es verdadera, pero encuentra resultados interesantes sobre la importancia de la estructura macroeconómica subyacente ya sea que esté en forma explícita o implícita.

\section{Modelos VAR}

\subsection{VAR estándar}

El modelo VAR estructural (Vectores Auto-regresivos Estructurales) parte de un sistema de ecuaciones simultáneas en forma estándar, donde todas las variables involucradas en el sistema son endógenas.

Siguiendo a Lütkepohl (1993), en la forma estándar del VAR todas las variables endógenas dependen de los rezagos de si misma y de los rezagos de las demás. Esta forma del VAR supone que el sistema no tiene componentes determinísticos y tiene una representación auto-regresiva de orden finito de la forma.

$$
\begin{gathered}
x_{t}=A_{1} x_{t-1}+A_{2} x_{t-2}+\ldots . .+A_{q} x_{t-q}+\varepsilon_{t} \\
e_{t} \rightarrow \text { i.i.d }\left(0, \Sigma_{e}\right)
\end{gathered}
$$

Donde $x_{t}$ es un vector $n \times 1$ de variables endógenas del sistema, $x_{t-i}$ son los vectores $n \times 1$ de las mismas variables endógenas rezagadas i periodos, $A_{i}$ son las matrices $n \times n$ de coeficientes.

En el sistema (12) los vectores autorregresivos pasan a la izquierda, de tal manera que los choques o innovaciones se pueden representar como:

$$
x_{t}-A_{1} x_{t-1}-A_{2} x_{t-2}-\ldots . .-A_{q} x_{t-q}=\varepsilon_{t}
$$


Que en términos del operador de rezagos se puede expresar de la siguiente manera:

$$
A(L) x_{t}=\varepsilon_{t} \leftrightarrow A(L)=I-A_{1} L-\ldots .-A_{q} L^{q}
$$

Donde $\mathrm{A}(\mathrm{L})$ es el operador de rezagos e I es la matriz identidad de $n \times n$

La representación VMA (Vector Moving Average) de (13) es:

$$
\begin{aligned}
x_{t} \leftrightarrow C(L) \varepsilon_{t} & \leftrightarrow C(L)=A(L)^{-1} \\
\leftrightarrow & \leftrightarrow(L)=I-C_{1} L-\ldots-C_{q} L^{q}
\end{aligned}
$$

Si C(L) es diferente de cero, entonces el modelo es estable y el VAR como sistema es estacionario.

\subsection{VAR estructural}

Según Amissano \& Giannini (1997) hay tres clases de VAR estructural, según la clase de restricciones que el investigador quiera implementar, relaciones contemporáneas entre las variables endógenas, restricciones de largo plazo o las dos. Para cada una de estas especificaciones hay un tipo de modelo VAR estructural: el modelo $\mathrm{K}$, el modelo $\mathrm{C}$ y el modelo A-B. En este trabajo se aplicará el modelo K, el cual tiene la siguiente especificación:

\section{Modelo K}

En este modelo $\mathrm{K}=\mathrm{Ao}$, es una matriz $\mathrm{n} \times \mathrm{n}$ e invertible en la cual están las relaciones contemporáneas entre las variables endógenas del sistema conocida como la matriz de coeficientes contemporáneos, de tal manera que se pueda pre-multiplicar al VAR estándar (13) por Ao, que es la matriz del lado izquierdo en (9).

La forma del VAR estructural es la siguiente:

$$
A_{0} A(L) x_{t}=A_{0} \varepsilon_{t}
$$

En la matriz Ao se encuentran las restricciones estructurales que imponen las relaciones contemporáneas entre variables económicas, pueden ser de modelos macroeconómicos de comportamiento o de modelos microeconómicos. En este trabajo la matriz Ao surge de las relaciones contemporáneas del modelo macroeconómico estructural $\mathrm{AD}-\mathrm{AS}$, anteriormente descrito.

Los choques o errores del VAR estructural(15) ahora se convierten en una combinación lineal de las restricciones impuestas en Ao y de los choques del VAR estándar $\varepsilon_{\mathrm{t} \text {, }}$ esto garantiza la ortogonalización de los choques del VAR estructural.

Los errores del VAR estructural son:

$$
\begin{aligned}
e_{t}=A_{0} \varepsilon_{t} & \leftrightarrow A_{0}^{-1} e_{t}=\varepsilon_{t} \leftrightarrow A_{0}^{-1} e_{t} e_{t}^{\prime}\left(A_{0}^{-1}\right)^{\prime}=\varepsilon_{t} \varepsilon_{t}^{\prime} \\
& \leftrightarrow A_{0}^{-1} e_{t} e_{t}^{\prime}\left(A_{0}^{-1}\right)^{\prime}=\Sigma_{\varepsilon} \\
& \leftrightarrow A_{0}^{-1} D\left(A_{0}^{-1}\right)^{\prime}=\Sigma_{\varepsilon}
\end{aligned}
$$

Donde $\mathrm{D}$ es una matriz identidad de $\mathrm{n} \times \mathrm{n}$, que en el caso del modelo macroeconómico AD-AS impuesto es $4 \times 4$. Por otro lado, $\Sigma \varepsilon$ es la matriz de varianzas y covarianzas de los errores del modelo no estructural (12).

A partir de (17) se calcula la matriz de coeficientes contemporáneos Ao, por el método de factorización conocido como descomposición de Choleski ${ }^{4}$, dada la información contenida en $\Sigma \varepsilon$.

Dado que $\Sigma \varepsilon$ tiene $n(n+1) / 2$ datos, entonces la matriz Ao debe tener $n(n+1) / 2$ restricciones no lineales y se deben dejar $n(n-1) / 2$ parámetros libres (ceros). Es necesario dejar $n(n-1) / 2$ parámetros libres porque de lo contrario podrían resultar varias matrices Ao al descomponer por Choleski (17). Una vez calculada la matriz Ao junto con los errores del VAR estándar en (12), se obtienen los errores del VAR estructural en (16).

\footnotetext{
4 Es la factorización más utilizada para matrices triangulares inferiores.
} 
A partir de los errores del VAR estructural y utilizando la representación VMA en (14) para el VAR estructural se obtienen los efectos de largo plazo a través de respuesta impulso, descomposición de varianza y descomposición histórica.

La contrastación teórica de las relaciones contemporáneas del modelo macroeconómico $\mathrm{AD}-\mathrm{AS}$ se analiza en la matriz Ao de coeficientes contemporáneos.

Los efectos de largo plazo de los choques de IS, AS, MP y LM se analizan con los resultados de la respuesta impulso y descomposición de varianza.

Las restricciones de corto plazo en la matriz Ao tienen incidencia en los efectos de largo plazo de los choques en el sistema. Esto diferencia los choques de un VAR estándar con los del VAR estructural.

Por otro lado, la descomposición o factorización de Choleski garantiza que la forma estructural tiene un vector de errores (innovaciones, choques) ortonormal. La descomposición de Choleski también asegura que se puedan identificar los choques en forma separada de IS, AS, MP o LM, a partir de choques en Y, P, R o M.

El VAR estructural (15) tiene la siguiente forma:

(18) $\left[\begin{array}{cccc}1 & 0 & a Y R & 0 \\ -a P Y & 1 & 0 & 0 \\ -a R Y & -a R P & 1 & 0 \\ -a M Y & -a M P & a M R & 1\end{array}\right] A(L)\left[\begin{array}{l}Y \\ P \\ R \\ M\end{array}\right]=\left[\begin{array}{cccc}1 & 0 & a Y R & 0 \\ -a P Y & 1 & 0 & 0 \\ -a R Y & -a R P & 1 & 0 \\ -a M Y & -a M P & a M R & 1\end{array}\right]\left[\begin{array}{l}\varepsilon_{I S, Y} \\ E_{A S P} \\ \varepsilon_{S P P, R} \\ E_{L M, M}\end{array}\right]$

Donde $A(L)=I-A_{1} L-\ldots-A_{q} L^{q}$ es la parte dinámica del VAR estándar.

Para que la descomposición de Choleski se pueda aplicar se debe imponer un cero en la matriz de coeficientes contemporáneos en (18), y para convertirla en una matriz triangular inferior la opción es hacer $\mathrm{aYR}=0$, esto supone que la tasa de interés $\mathrm{R}$ no tiene efectos contemporáneos sobre el PIB real. Como resultado surge el siguiente sistema VAR estructuraldinámico:

$$
\begin{aligned}
& {\left[\begin{array}{cccc}
1 & 0 & 0 & 0 \\
-a_{P Y} & 1 & 0 & 0 \\
-a_{R Y} & -a_{R P} & 1 & 0 \\
-a_{M Y} & -a M P & a_{M R} & 1
\end{array}\right] A(L)\left[\begin{array}{c}
Y \\
P \\
R \\
M
\end{array}\right]=\left[\begin{array}{cccc}
1 & 0 & 0 & 0 \\
-a_{P Y} & 1 & 0 & 0 \\
-a_{R Y} & -a_{R P} & 1 & 0 \\
-a_{M Y} & -a_{M P} & a_{M R} & 1
\end{array}\right]\left[\begin{array}{c}
\varepsilon_{I S, Y} \\
\varepsilon_{A S, P} \\
\varepsilon_{M P, R} \\
\varepsilon_{L M, M}
\end{array}\right]} \\
& \leftrightarrow \quad A_{0} A(L) x_{t}=A_{0} \varepsilon_{t}
\end{aligned}
$$

La matriz triangular Ao es conocida en la literatura de vares estructurales como "Wold Causal Chain". Lo que diferencia el VAR estructural del var estándar es que por (16) $e_{t}=A_{0} e_{t}$, dado que los errores del VAR estructural $\left(e_{t}\right)$ son una combinación lineal de los errores del var estándar $\left(\varepsilon_{t}\right)$ en (12) y de las restricciones de corto plazo que impone la matriz de coeficientes contemporáneos Ao en (19).

De tal manera que:

$$
\begin{array}{ll}
\text { IS... } & e_{I S, Y}=\varepsilon_{I S, Y} \\
\text { AS... } & e_{A S, P}=-a P Y \varepsilon_{I S, Y}+\varepsilon_{A S, P} \\
\text { MP... } & e_{M P, R}=-a R Y \varepsilon_{I S, Y}-a R P \varepsilon_{A S, P}+\varepsilon_{M P, R} \\
\text { LM... } & e_{L M, M}=-a M Y \varepsilon_{I S, Y}-a M P \varepsilon_{A S, P}+a M R \varepsilon_{M P, R}+\varepsilon_{L M, M}
\end{array}
$$

Los choques, innovaciones o errores del VAR estructuralen (20) son la clave para calcular los efectos dinámicos de políticas monetarias (MP), de oferta agregada (AS) o de LM sobre las variables del sistema. El paso previo para calcular los errores del VAR estructuralen (20) es calcular la matriz de coeficientes contemporáneos Ao y una vez calculada ésta matriz se puede hacer el análisis dinámico de los choques de política monetaria, a través de respuesta impulso y descomposición de varianza.

La matriz de coeficientes contemporáneos Ao tiene $\mathrm{n}(\mathrm{n}+1) / 2$ restricciones no lineales y $n(n-1) / 2$ parámetros libres, entonces se puede factorizar (17) por Choleski ${ }^{5}$.

Por otro lado, se discute en la literatura de VAR estructurales si las variables endógenas tienen que ser estacionarias individualmente; es decir, si deben estar en logaritmos, en diferencias de logaritmos o en niveles.

5 Dado que la matriz Ao en (19) es triangular inferior y dado el orden específico de las series, la descomposición de Choleski de la matriz de varianzas y covarianzas es única, cuestión que no se garantiza si la matriz no es triangular. 
La recomendación en la literatura de VAR estructural ${ }^{6}$ $e$ inclusive en los paquetes econométricos que calculan los Rats y Stata por ejemplo, es que se pruebe con diferentes especificaciones cada modelo, en este caso el AD-AS. En últimas, lo que interesa en cada especificación, es que el modelo sea estable, lo que implica que el sistema VAR estructural estacionario. Cuando se corren las diferentes especificaciones y se calculan las matrices contemporáneas, el hecho de que el modelo converja significa que el modelo es globalmente estable, lo que a su vez implica que el sistema VAR estructural estacionario ${ }^{7}$.

El número y características de las diferentes especificaciones dependen en muchos casos del conocimiento del investigador de la estructura macroeconómica subyacente y del comportamiento individual de cada serie. También depende mucho de la forma como el investigador quiera mostrar los resultados tanto en las relaciones contemporáneas como en el largo plazo.

Se hicieron tres pruebas para conocer el número de rezagos en $A(L)$ : la prueba de 4 versus 8 rezagos, la prueba Akaike Information Criterion (AIC) y la prueba de Schwarts Bayesian Criterion $(\mathrm{SBC})^{8}$.

\section{Especificación de las series}

Las diferentes especificaciones del modelo AD-AS que se corren están con datos trimestrales para Colombia entre el segundo trimestre de $1995^{\circ}$ y el segundo trimestre de 2011 de las variables PIB real (Y), inflación $(\mathrm{P})$, tasa de intervención de política monetaria del Banco de la República $(\mathrm{R})^{10}$, y como medios de pago se utilizan cuatro variables: la base monetarias, M1, M2 y M2 + CDT, todas en términos reales (M).
Las cuatro variables que se utilizan como medios de pago y el Índice de Precios al Consumidor (IPC) se des-estacionalizaron utilizando el suavizado que mejor se ajustó a los datos. ${ }^{11}$ Se empalmaron las series del pib base 1994 con las del PIB base 2005.

Se tomaron los datos de la tasa de intervención del Banco de la República vigentes a finales de cada trimestre, aunque las políticas contractivas o expansivas no necesariamente coincidieran con el final de cada trimestre.

Se discute en la literatura de VAR sobre cuál es la mejor especificación en la cual los datos deben estar. Se recomienda que los datos estén en logaritmos, en diferencias de logaritmos, en tasas de crecimiento o en variaciones de tasas. En la literatura de modelos VAR se recomienda que las variables involucradas sean estacionarias para asegurar que la especificación de cada modelo sea estable y por lo tanto estacionario como sistema.

Para escoger la especificación correcta en la cual deben estar las variables utilizadas como medios de pago, se grafican las series base monetaria, M1, M2 y M2+CDT, todas en diferencias de logaritmos, en la Ilustración 1. El análisis gráfico de estas series indica que las pruebas de estacionariedad a utilizar deben tener constante pero no tendencia, dado que las segundas diferencias de los logaritmos tienen constante cero (no se muestran en el Ilustracion), lo que indica que la prueba no debe tener componente tendencial, pero si constante porque las primeras diferencias tienen medias diferentes de cero. Se hicieron dos pruebas de estacionariedad: Dickey Fuller aumentado y Phillips Perron. ${ }^{12}$

\footnotetext{
A manera de ejercicio académico se prueban diferentes especificaciones para la matriz de coeficientes contemporáneos.

En modelos VAR la estabilidad implica estacionariedad, pero lo inverso no es siempre cierto.

8 El número de rezagos obtenidos en estas pruebas sirven de referencia al adicionar los rezagos en diferencias en las pruebas de raiz unitaria de Dickey Fuller Aumentada y Phillips Perron.

9 Las series históricas se tomaron de las bases de datos del Banco de la República.

10 Se toman datos desde 1995 debido a que el Banco de la República comienza a utilizar la tasa de intervención como herramienta de política monetaria desde 1995.

11 Se utilizó el comando smooth de Stata, que elige la mejor especificación en tendencia: lineal o exponencial y la mejor según la estacionalidad: aditiva o multiplicativa.

12 Se utilizaron las diferencias con dos rezagos en la prueba de Dickey Fuller aumentada y dos rezagos en la prueba de Phillips Perron.
} 
Ilustración 1. Series de Base monetaria ${ }^{13}$.

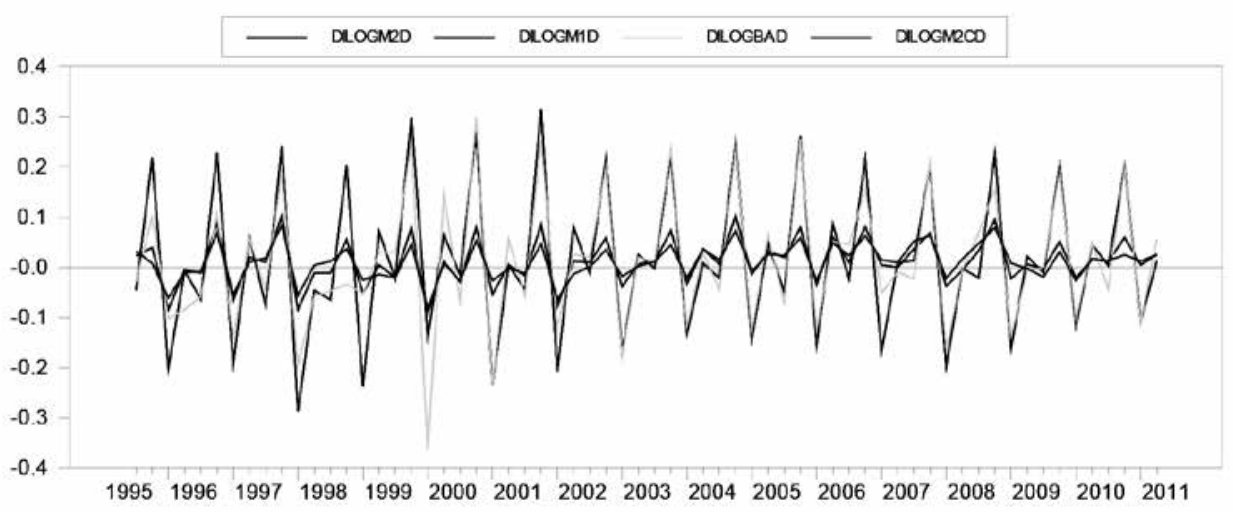

Los resultados de las pruebas tanto de Dickey Fuller aumentada como de Phillips Perron indican que las series base monetaria, M1, M2 y M2+CDT en diferencias de logaritmos son estacionarias ${ }^{14}$ con constante y sin tendencia, con un nivel de significancia del 5\%. Las mismas series en logaritmos tienen raíz unitaria, por lo tanto son I(1). Según los resultados de las pruebas de estacionariedad la mejor especificación de las variables utilizadas como medios de pago es en diferencias de logaritmos.

Para analizar la especificación del IPC y del PIB se presenta la Ilustración 2 , en el cual se grafica la diferencia del logaritmo del IPC y la diferencia del logaritmo del PIB. Gráficamente se puede ver que las pruebas de raíz unitaria deben tener constante pero no tendencia.

Según las pruebas de Dickey Fuller aumentada (DFA) y Phillips Perron $(\mathrm{PP})^{15}$, las dos series: diferencia del logaritmo del IPC y diferencia del logaritmo del PIB son estacionarias con constante y sin tendencia, con un nivel de significancia del 5\%, y las diferencias de dos rezagos adicionales. Esto indica que se deben utilizar las dos series en diferencias de logaritmos, en las diferentes especificaciones de los modelos VAR estructurales. Las series tasa de interés de intervención del Banco de la República y diferencias de la tasa de intervención se presentan en la Ilustración 3.

La serie tasa de interés en niveles es estacionaria en la prueba de DFA con un $10 \%$ de significancia y en la prueba de PP con una significancia del 5\%. Las dos pruebas se corrieron con constante y sin tendencia y con las diferencias de dos rezagos adicionales. Por otro lado, la serie diferencias de la tasa de intervención es estacionaria tanto en la prueba de DFA como en la de PP, con una significancia del 5\%, la cual se corrió con constante y sin tendencia y las diferencias de dos rezagos adicionales.

\section{Especificación de los modelos}

Se corrieron ocho especificaciones del modelo AD$\mathrm{AS}^{16}$, es decir, de la matriz de coeficientes contemporáneos Ao en (19). Cuatro de los modelos se corrieron con las diferencias del logaritmo del PIB, las diferencias del PIC y la diferencia de la tasa de intervención con cada una de las variables que se utilizaron como medios de pago: las diferencias de

\footnotetext{
Calculo de los autores.

Ver anexo 2.

Ver anexo 2.

Se utilizó la metodología svar en Stata, con la especificación para un modelo con matriz de restricciones contemporáneas.

Se utiliza la metodología de factorización de Choleski en Stata, con las opciones para factorizar la matriz de coeficientes contemporáneos por medio de la metodología de Broyden, Fletcher, Goldfarb y Shanno (BFGS), que es la única que calcula errores estándar.
} 
Ilustración 2. Especificación del IPC y del PIB ${ }^{18}$.

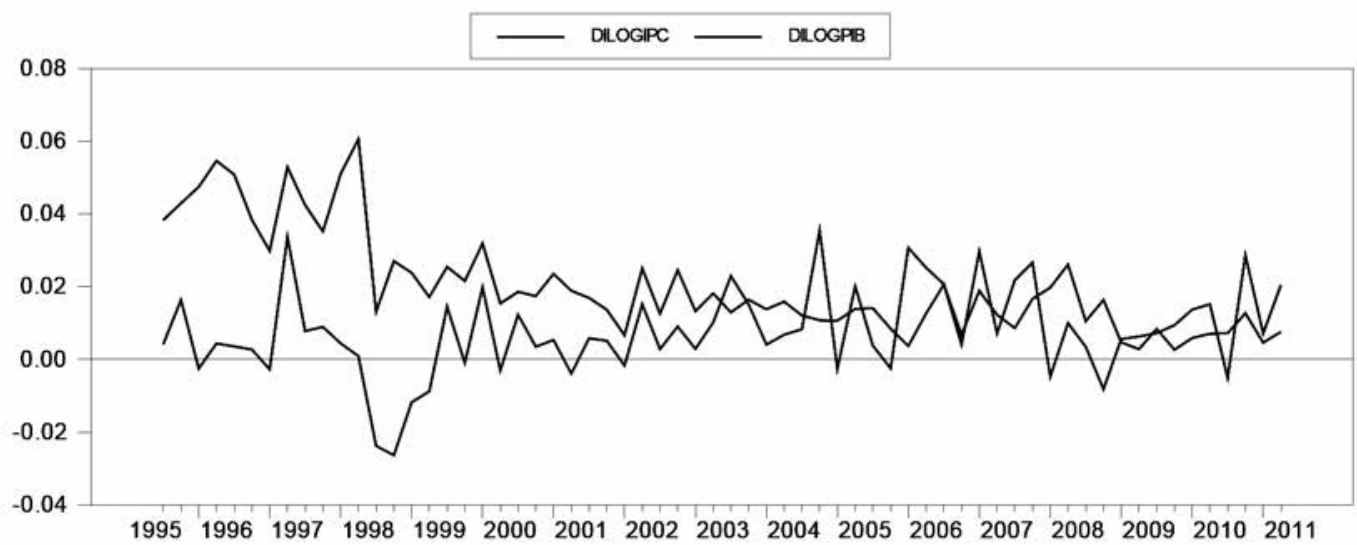

Ilustración 3. Tasa de interés de intervención del Banco de la República ${ }^{19}$.

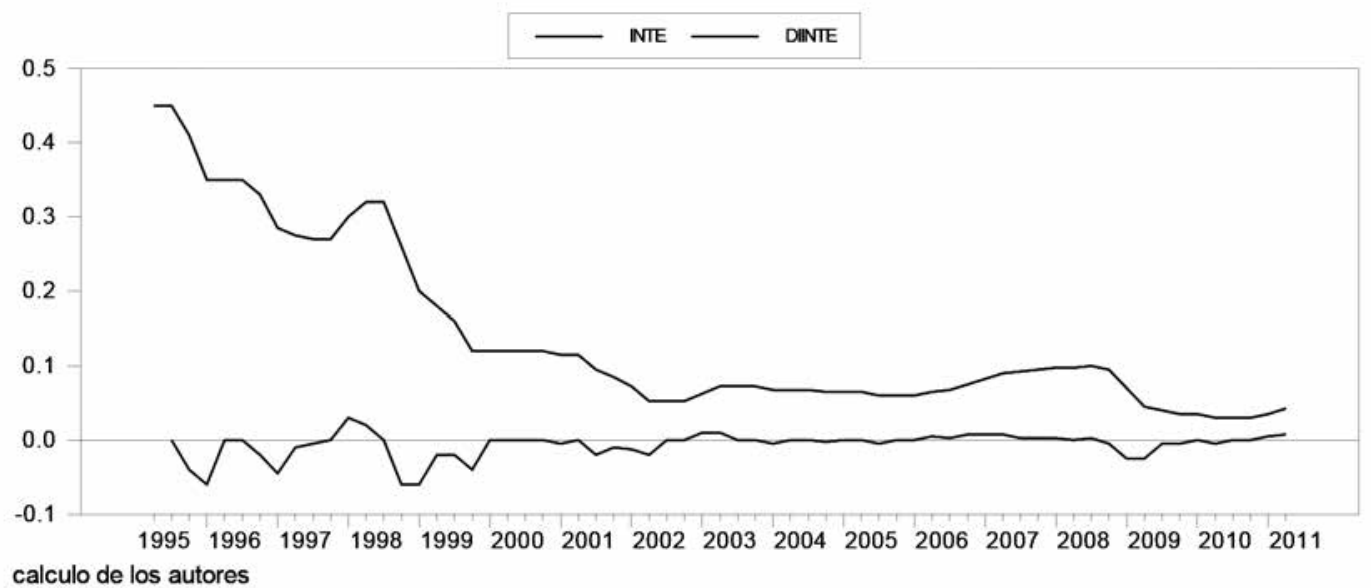

los logaritmos de la base monetaria, de M1, de M2 y de M2+CDT, y los otros cuatro modelos se corrieron cambiando las diferencias de la tasa de intervención por la misma pero en niveles. Cada una de las especificaciones se corrió con el número de rezagos que las pruebas: Akaike Information Criterion, HannanQuinn Information Criterion y Schwarts Bayesian Criterion $^{20}$, indican.
Los parámetros o coeficientes de las diferentes especificaciones de los modelos se interpretan como elasticidades dado que las variables están en diferencias de logaritmos, variaciones de tasas o tasas en niveles.

18 Cálculo de los autores.

19 Cálculo de los autores.

20 En el anexo 3 se presenta un resumen de las pruebas para el número óptimo de rezagos. 


\section{Resultados}

En la tabla 1 se presentan las diferentes matrices de coeficientes contemporáneos Ao de (19), de acuerdo a las diferentes especificaciones, calculadas según la factorización de Choleski ${ }^{20}$ en (17). En la Tabla 1, se presentan las matrices de coeficientes contemporáneos bajo ocho especificaciones. En la parte superior de cada matriz se presenta la especificación de las series que componen cada modelo, el número de rezagos utilizados para que la descomposición de
Choleski converja, así como el número de iteraciones utilizadas para la convergencia.

En todos los modelos se utiliza la diferencia del logaritmo del PIB y del IPC. En los modelos 1 y 2 se utiliza la diferencia del logaritmo de M2+CDT. En los modelos 3 y 4 se utiliza la diferencia del logaritmo de la base monetaria. Los modelos 5 y 6 se corren con la diferencia del logaritmo de M1 y en los modelos 7 y 8 se utiliza la diferencia del logaritmo de M2.

Tabla 1. Coeficientes contemporáneos del modelo AD-AS ${ }^{21}$.

\begin{tabular}{|c|c|c|c|c|c|c|c|}
\hline \multicolumn{4}{|c|}{$\begin{array}{l}\text { 1.Variables:d(logpib), dilogipc, dinte, } d(\text { logm2c) } \\
\text { Rezagos: } 4 \text { Convergencia en } 5 \text { iteraciones }\end{array}$} & \multicolumn{4}{|c|}{$\begin{array}{l}\text { 2.Variables: } d(\text { logpib), dilogipc, inte, } d(\text { logm2c) } \\
\text { Rezagos: } 1 \text { Convergencia en } 6 \text { iteraciones }\end{array}$} \\
\hline 1.0000 & 0.0000 & 0.0000 & 0.0000 & 1.0000 & 0.0000 & 0.0000 & 0.0000 \\
\hline-.08264 & 1.0000 & 0.0000 & 0.0000 & -.1036 & 1.0000 & 0.0000 & 0.0000 \\
\hline-.19471 & $-.3316^{\star}$ & 1.0000 & 0.0000 & -.1049 & $-.5310^{*}$ & 1.0000 & 0.0000 \\
\hline$-.3543^{\star}$ & $1.1615^{\star}$ & .0267 & 1.0000 & $-.3774^{\star}$ & $1.4816^{*}$ & $-.0158^{*}$ & 1.0000 \\
\hline \multicolumn{4}{|c|}{$\begin{array}{l}\text { 3.Variables: } d(\text { (logpib), dilogipc, dinte, } d(\text { logba) } \\
\text { Rezagos: } 1 \text { Convergencia en } 8 \text { iteraciones }\end{array}$} & \multicolumn{4}{|c|}{$\begin{array}{l}\text { 4.Variables: } d(\text { logpib), dilogipc, inte, d(logba) } \\
\text { Rezagos: } 1 \text { Convergencia en } 5 \text { iteraciones }\end{array}$} \\
\hline 1.0000 & 0.0000 & 0.0000 & 0.0000 & 1.0000 & 0.0000 & 0.0000 & 0.0000 \\
\hline-.07044 & 1.0000 & 0.0000 & 0.0000 & -.1082 & 1.0000 & 0.0000 & 0.0000 \\
\hline-.1187 & $-.5134^{\star}$ & 1.0000 & 0.0000 & -.1110 & $-.4779 *$ & 1.0000 & 0.0000 \\
\hline$-1.7047^{*}$ & $4.2354^{*}$ & $-.3156^{\star}$ & 1.0000 & $-1.6006^{*}$ & $2.3032^{*}$ & $-.05937^{\star}$ & 1.0000 \\
\hline \multicolumn{4}{|c|}{$\begin{array}{l}\text { 5.Variables: } d(\text { logpib), dilogipc, dinte, } d(\operatorname{logm} 1) \\
\text { Rezagos: } 1 \text { Convergencia en } 7 \text { iteraciones }\end{array}$} & \multicolumn{4}{|c|}{$\begin{array}{l}\text { 6.Variables: } d(\text { logpib), dilogipc, inte, } d(\operatorname{logm} 1) \\
\text { Rezagos: } 2 \text { Convergencia en } 5 \text { iteraciones }\end{array}$} \\
\hline 1.0000 & 0.0000 & 0.0000 & 0.0000 & 1.0000 & 0.0000 & 0.0000 & 0.0000 \\
\hline-.01021 & 1.0000 & 0.0000 & 0.0000 & -.07980 & 1.0000 & 0.0000 & 0.0000 \\
\hline-.0941 & $-.5334^{*}$ & 1.0000 & 0.0000 & -.0824 & $-.6102^{*}$ & 1.0000 & 0.0000 \\
\hline$-1.1599^{*}$ & $2.2391^{*}$ & -.20343 & 1.0000 & $-1.2530^{*}$ & $2.0904^{*}$ & $-.01159^{*}$ & 1.0000 \\
\hline \multicolumn{4}{|c|}{$\begin{array}{l}\text { 7.Variables: } d(\text { logpib), dilogipc, dinte, } d(\operatorname{logm} 2) \\
\text { Rezagos: } 1 \text { Convergencia en } 6 \text { iteraciones }\end{array}$} & \multicolumn{4}{|c|}{$\begin{array}{l}\text { 8.Variables: } d(\text { logpib), dilogipc, inte, } d(\operatorname{logm} 2) \\
\text { Rezagos: } 1 \text { Convergencia en } 7 \text { iteraciones }\end{array}$} \\
\hline 1.0000 & 0.0000 & 0.0000 & 0.0000 & 1.0000 & 0.0000 & 0.0000 & 0.0000 \\
\hline-.0976 & 1.0000 & 0.0000 & 0.0000 & -.1320 & 1.0000 & 0.0000 & 0.0000 \\
\hline-.1779 & $-.3858^{*}$ & 1.0000 & 0.0000 & -.1510 & $-.4730^{\star}$ & 1.0000 & 0.0000 \\
\hline$-.5606^{\star}$ & $1.3072^{*}$ & $-.0317^{\star}$ & 1.0000 & $-.6066^{*}$ & $1.1824^{*}$ & $-.0177^{*}$ & 1.0000 \\
\hline
\end{tabular}

21 Cálculo de los autores utilizando STATA. En el anexo 4 se presenta la salida en Stata de la matriz de coeficientes contemporáneos del modelo1. Los ocho modelos son estables, por lo tanto estacionarios. Los coeficientes marcados con (*) son significativos al 95\%. Los coeficientes marcados con (') son significativos al 90\%. Los coeficientes en negrilla tienen el signo esperado según el modelo AD-AS de comportamiento. 
Los modelos $1,3,5$ y 7 se corren con las diferencias de la tasa de intervención y el 2, 4, 6 y 8 con la tasa de intervención en niveles. En el anexo 4 se presenta la salida de una de las especificaciones de la matriz de coeficientes contemporáneos. La convergencia (factorización) de las matrices contemporáneas se calculó con el método "Genetic" para luego utilizar el método "BFGS" el único en el cual se presentan los errores estándar y la significancia de los coeficientes contemporáneos. En las ocho especificaciones hubo convergencia, lo que indica que todas las especificaciones son estables y, por lo tanto, todos los ocho VAR estructural corridos son estacionarios como sistema.

\subsection{Análisis contemporáneo}

Los resultados de la tabla 10 conforman el sistema de ecuaciones en (6), (7) y (8). Las relaciones contemporáneas entre las variables endógenas del sistema se pueden entender mejor al pasar los coeficientes de las variables de la tabla 1 , a la derecha, en cada uno de los modelos. Las curvas de oferta agregada (AS), la LM y la MP (política monetaria) de los modelos calculados son las siguientes:

\section{Modelo 1}

$$
\begin{aligned}
& P=0,08264 Y \varepsilon_{A S, P} \\
& R=0,3316 \mathbf{P}+0,1947 \mathrm{Y}+\varepsilon_{M P, R} \\
& M=-0,0267 \mathbf{R}-1,1615 P^{*}+0,3573 Y^{*}+\varepsilon_{L M, M}
\end{aligned}
$$

\section{Modelo 2}

$$
\begin{aligned}
& P=0,1036 Y+\varepsilon_{A S, P} \\
& R=0,531 \mathrm{P}^{*}+0,1049 Y+\varepsilon_{M P, R} \\
& M=0,0158 R-1,4816 P^{*}+0,3774 Y^{*}+\varepsilon_{L M, M}
\end{aligned}
$$

\section{Modelo 3}

$$
\begin{aligned}
& P=0,0704 Y+\varepsilon_{A S, P} \\
& R=0,5134 \mathbf{P}+0,1187 Y+\varepsilon_{M P, R} \\
& M=0,3156 R^{*}-4,2354 P^{*}+1,7047 Y^{*}+\varepsilon_{L M, M}
\end{aligned}
$$

Modelo 4

$$
\begin{array}{lc}
P=0,1082 Y+\varepsilon_{A S, P} & \text { AS } \\
R=0,4779 \mathrm{P}+0,1110 \mathrm{Y}+\varepsilon_{M P, R} & \text { MP } \\
M=-0,5937 \mathrm{R}-2,2032 P^{*}+1,6006 \mathrm{Y}^{*}+\varepsilon_{L M, M} \text { LM }
\end{array}
$$

Modelo 5

$$
\begin{array}{ll}
P=0,1021 \mathrm{Y}+\varepsilon_{A S, P} & \text { AS } \\
R=0,5334 \mathrm{P}+0,0941 \mathrm{Y}+\varepsilon_{M P, R} & \text { MP } \\
M=-0,2034 \mathrm{R}-2,2391 P^{*}+\mathbf{1}, 1599 Y^{*}+\varepsilon_{L M, M} & \text { LM }
\end{array}
$$

\section{Modelo 6}

$$
\begin{array}{ll}
P=0,0798 \mathrm{Y}+\varepsilon_{A S, P} & \text { AS } \\
R=0,6102 \mathrm{P}+0,0824 \mathrm{Y}+\varepsilon_{M P, R} & \text { MP } \\
M=-0,0115 \mathrm{R}-2,0924 P^{*}+1,2530 \mathrm{Y}^{*}+\varepsilon_{L M, M} & \text { LM }
\end{array}
$$

\section{Modelo 7}

$$
\begin{array}{ll}
P=0,0976 Y+\varepsilon_{A S, P} & \text { AS } \\
R=0,3858 \mathrm{P}+0,1779 Y+\varepsilon_{M P, R} & \text { MP } \\
M=0,0317 R-1,3072 P^{*}+0,5606 Y^{*}+\varepsilon_{L M, M} & \text { LM }
\end{array}
$$

\section{Modelo 8}

$$
\begin{array}{ll}
P=0,1320 \mathrm{Y}+\varepsilon_{A S, P} & \text { AS } \\
R=0,4730 \mathrm{P}+0,1510 \mathrm{Y}+\varepsilon_{M P, R} & \mathrm{MP} \\
M=0,0177 R-1,1824 P^{*}+0,60666 \mathrm{Y}+\varepsilon_{L M, M} & \mathrm{LM}
\end{array}
$$

Observando los resultados de los ocho modelos, teniendo en cuenta la significancia entre las relaciones contemporáneas de las variables (*) y los signos esperados contemporáneos (en negrilla), se puede hacer el siguiente análisis en las relaciones contemporáneas de la curva de oferta agregada (AS), de la curva LM y de las relaciones de política monetaria (MP).

\subsubsection{Curva de Oferta Agregada (AS)}

De los resultados de la tabla 1 se puede decir que en las ocho especificaciones hay una relación posi- 
tiva contemporánea en el plano P-Y, es decir, entre el crecimiento económico y la variación de la inflación $\left(\mathrm{a}_{\mathrm{PY}}\right.$ en 19). Se comprueba la relación positiva que la teoría económica y el modelo AD-AS anticipan de la curva de oferta agregada (AS en 2'). Sin embargo, en ninguna de las ocho especificaciones el coeficiente es significativo con una confiabilidad del 95\%. Esto indica que el signo es el esperado en el plano P-Y, pero, siendo rigurosos la evidencia no confirma lo que se anticipa en (2') sobre la relación positiva y significativa en el plano P-Y, por el contrario los resultados indican que la pendiente es cero entre el crecimiento y la inflación. Los datos para Colombia indican que los precios son rígidos en el corto plazo, lo que está de acuerdo con las teorías keynesianas, que afirman que la curva de oferta agregada tiene pendiente cero en el corto plazo. Esto lo que significa es que, en términos contemporáneos, el crecimiento económico no influye sobre la inflación en Colombia.

\subsubsection{Curva LM}

De la curva LM se puede decir que en las ocho especificaciones hay una relación negativa contemporánea entre la variación de la inflación y la tasa de crecimiento del dinero. Las ocho especificaciones son significativas al 95\% de confiabilidad. Se contradice entonces la teórica relación positiva que el modelo AD-AS anticipa para la curva LM en (4'). Esto indica que por lo menos en términos contemporáneos un aumento de la inflación disminuye la tasa de crecimiento del dinero. Existe una relación contemporánea negativa y significativa en el hiperplano M-P, es decir la pendiente no es cero. Lo que se puede afirmar con los resultados es que, por lo menos en términos contemporáneos, las variaciones de la inflación y del dinero van en sentido contrario en Colombia.

En la misma curva LM y, en las ocho especificaciones, hay una relación contemporánea positiva entre el crecimiento económico y la variación de la cantidad de dinero. Esto está de acuerdo con la teoría económica, además en todas las especificaciones esta relación es significativa y positiva con una confiabilidad del $95 \%$. Lo que indica esto es que a mayor crecimiento económico mayor crecimiento del dinero en términos contemporáneos, es decir, una relación positiva en el hiperplano M-Y, que está de acuerdo a (4').

Por otro lado, en el hiperplano M-R en (19), en cuatro de las ocho especificaciones hay una relación negativa y contemporánea entre la tasa de intervención del Banco de la República y el crecimiento de la cantidad de dinero. Sin embargo, solo cuando se utiliza la base monetaria como medio de pago el coeficiente es significativo con el $95 \%$ de confiabilidad. En los cuatro modelos en los cuales esta relación es positiva no son significativos los coeficientes.

Lo que significa lo anterior es que los resultados para Colombia indican que no hay una relación negativa contemporánea entre la variación de la tasa de intervención del Banco de la República y el crecimiento de la cantidad de dinero (el coeficiente es cero), o en otras palabras, no se cumple la relación contemporánea que anticipa el modelo AD-AS en (4').

Por último, en términos contemporáneos hay una relación negativa y significativa entre la inflación y la cantidad de dinero. Lo cual está de acuerdo a lo esperado bajo el modelo AD-AS en (4'). Sin embargo, en todos los modelos la cantidad de dinero disminuye a tasas por encima del aumento de la inflación, una respuesta contra intuitiva del dinero frente a la inflación en términos contemporáneos.

\subsubsection{Política monetaria (MP)}

En las ocho especificaciones hay una relación contemporánea positiva entre el crecimiento económico y la variación de la tasa de intervención del Banco de la República. Sin embargo, en ninguna de las especificaciones el coeficiente es significativo con un nivel del $95 \%$. Se puede afirmar, que no hay una relación contemporánea entre el crecimiento económico y los cambios de la tasa de intervención en el hiperplano R-Y ( $\mathrm{a}_{\mathrm{RY}}$ en 19). En otras palabras, hay una relación contemporánea positiva entre el crecimiento económico y la tasa de interés de intervención tanto en niveles como en diferencias. Esto se puede explicar por el hecho de que la variable objetivo tanto de corto plazo como de largo plazo es la inflación y no el crecimiento económico. 
Por otro lado, hay una relación positiva y significativa al $95 \%$ entre la variación de la inflación y la tasa de intervención del Banco de la República tanto en niveles de la tasa de intervención como en diferencias. En el hiperplano R-Y se cumple el signo esperado en (3').

Lo anterior es lógico dado que la variable objetivo del Banco de la República es la inflación, tanto en el corto como en el largo plazo y, por otro lado, la variable instrumento es la tasa de intervención. Entonces los aumentos de la tasa de intervención responden a aumentos de la inflación o viceversa.

\subsection{Efectos dinámicos de choques de política monetaria}

Para analizar los efectos dinámicos de choques de política monetaria se utilizan dos herramientas econométricas: respuesta impulso y descomposición de varianza. En las metodologías conocidas como respuesta impulso y descomposición de varianza, se le da un choque de una desviación estándar a la variable tasa de intervención del Banco de la República. Técnicamente es aumentar $\varepsilon_{\mathrm{MPR}}$ en una unidad en la MP de los ocho modelos anteriores, y se analizan los efectos dinámicos posteriores sobre la inflación y el crecimiento económico.

La respuesta impulso calcula los efectos posteriores no acumulados en cada una de las variables respuesta (inflación y crecimiento), mientras que la descomposición de varianza calcula el porcentaje acumulado de cambio de cada una de estas variables ante los choques de política monetaria vía tasa de intervención.

\subsubsection{Respuesta impulso}

Los efectos dinámicos de choques de política monetaria sobre las variables endógenas en el modelo AD-AS en (11) ${ }^{22}$, se analizan con la respuesta impulso que el modelo VAR estructural arroja, dadas las restricciones de corto plazo de las matrices contemporáneas de la tabla 1. Otra manera de entender los efectos dinámicos de política monetaria es suponiendo que en los ocho modelos anteriores y en la curva $\mathrm{MP}$, se aumenta $\varepsilon_{\mathrm{MPR}}$ en una desviación estándar y se analiza el efecto dinámico sobre las variables $\mathrm{Y}$ (crecimiento económico) y $\mathrm{P}$ (inflación).

En la ilustración 4, se presenta la respuesta impulso de un aumento de una desviación estándar en la tasa de intervención del Banco de la República sobre las series crecimiento económico y variación de la inflación. En el eje horizontal de cada modelo se presenta la evolución dinámica de las variables hasta 10 trimestres y en el eje vertical está la respuesta de las variables inflación y crecimiento económico, debido al choque de la tasa de intervención. Además cada modelo presenta los intervalos de confianza del $95 \%$ alrededor de la respuesta al choque.

De la respuesta impulso de la ilustración 4 se puede analizar lo siguiente: ante un aumento de una desviación estándar en la tasa de intervención del Banco de la República (en niveles) no hay efectos sobre la inflación. En tres de los cuatro modelos el cero se mantiene dentro de los intervalos de confianza (95\%), lo cual indica que las políticas monetarias vía aumentos de la tasa de intervención en niveles no tienen efectos sobre la inflación. Solamente cuando se utiliza M1 como medio de pago el choque positivo de política monetaria tiene efectos negativos y significativos sobre la inflación en el tercero y séptimo trimestres después del choque.

Cuando se utiliza la tasa de intervención en diferencias, ante un choque positivo de ésta, hay efectos negativos y significativos sobre la inflación, pero estos son cíclicos. En todos los casos el efecto negativo y significativo se presenta entre el segundo y tercer trimestre y luego entre el sexto y octavo trimestre. Se cumple lo que se anticipa la estática comparativa en (11), pero los efectos son cíclicos.

Sobre los efectos en el crecimiento real de la economía ante un choque de la tasa de intervención, tanto en niveles como en diferencias, se puede decir que en todos los casos hay un efecto negativo que se

22 Se utilizó el comando irf en Stata para cada una de las especificaciones de la tabla 1. 
Ilustración 4. Efectos de choques de política monetaria del Banco de la República²3.

\section{Modelo 1}

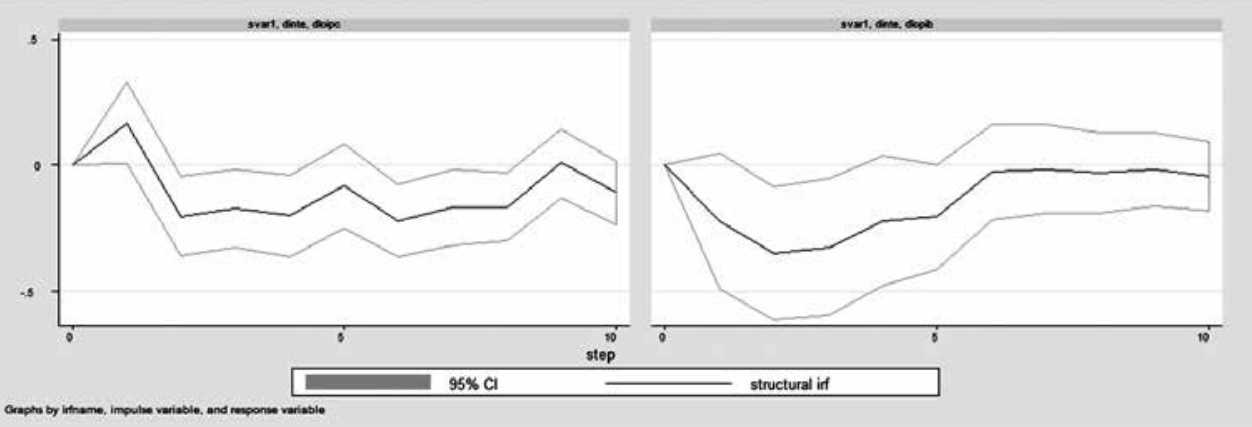

\section{Modelo 2}

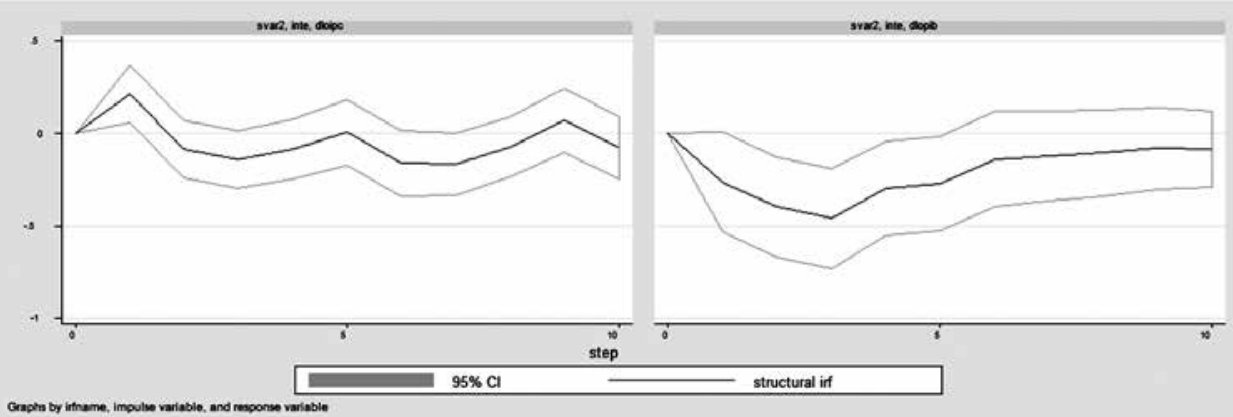

Modelo 3

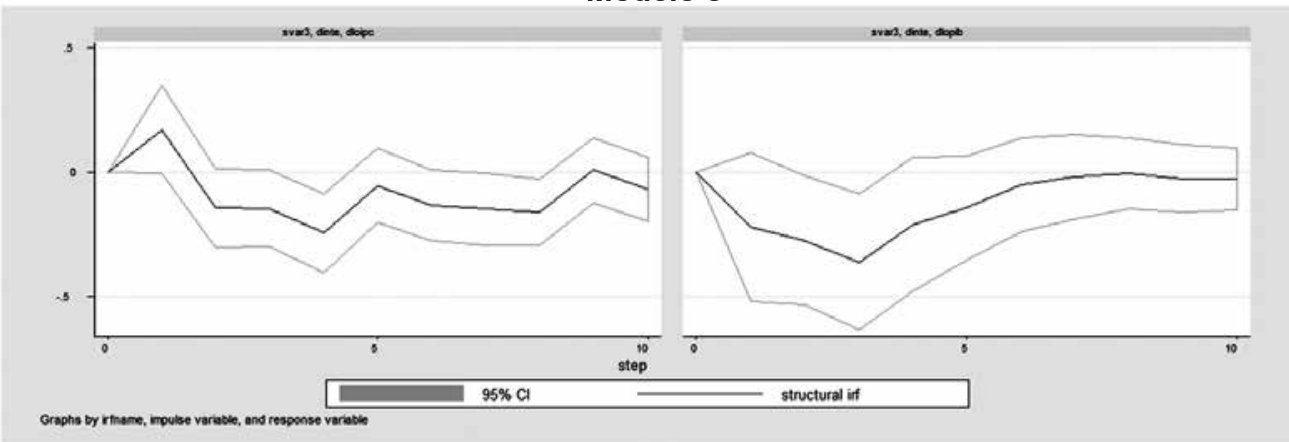

Modelo 4

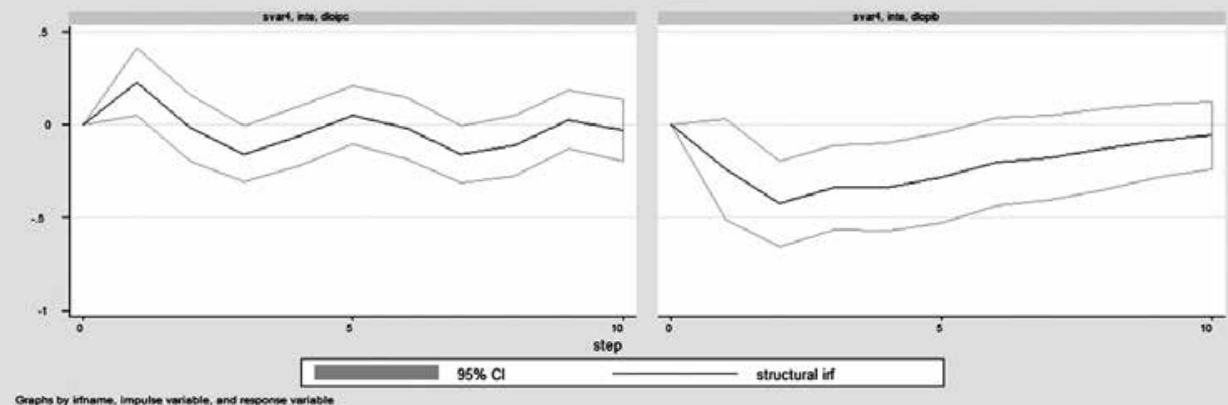

Cálculos de los autores en Stata. 


\section{Modelo 5}
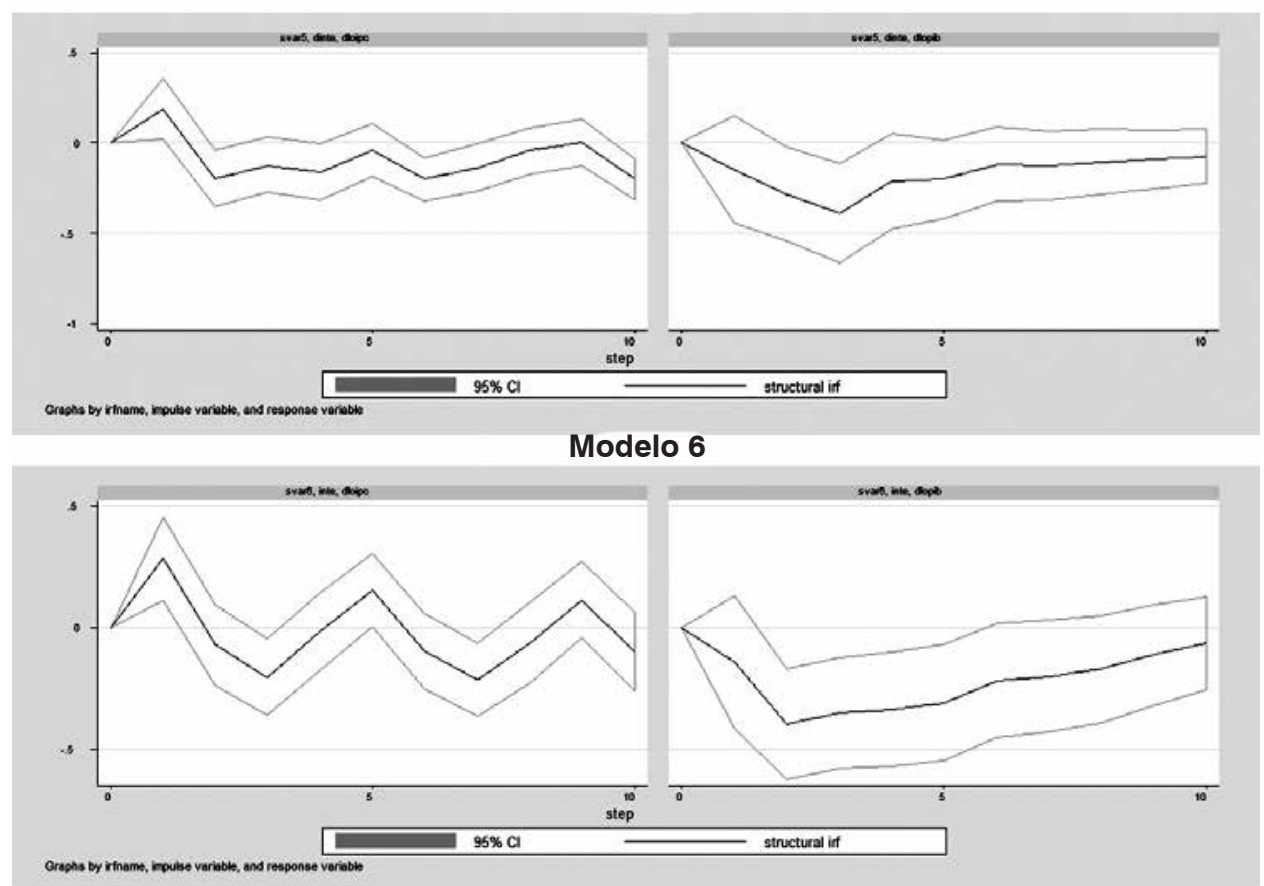

Modelo 7

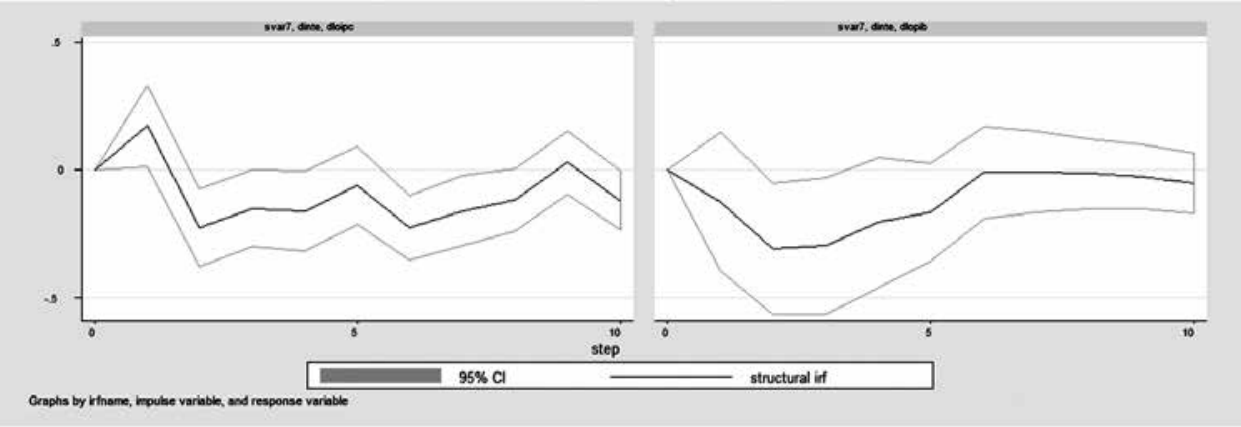

Modelo 8

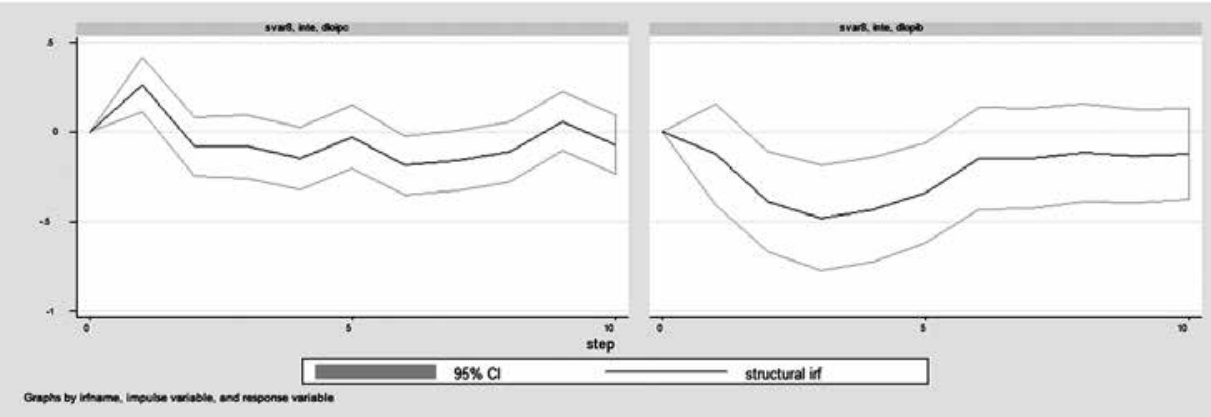


presenta entre 2 y 3 trimestres después del choque. Se cumple lo que se anticipa en (11). Los intervalos de confianza en los ocho modelos permanecen por debajo de cero entre el segundo y tercer trimestres, lo que indica que la disminución en el crecimiento económico ante choques positivos de la tasa de intervención, son significativos.

\subsubsection{Descomposición de varianza}

Los efectos dinámicos acumulados ante choques de política monetaria vía tasa de intervención sobre el crecimiento económico y la inflación, están tabulados en las siguientes tablas.

Se presentan las tablas de choques cuando la variable utilizada es M2 + CDT, las otras tablas se presentan como anexos al final del trabajo.

De los resultados encontrados con la descomposición de varianza en los ocho modelos (SVAR1 a SVAR8), se puede decir que un choque positivo de la tasa de intervención en niveles (modelos 2, 4, 6 y 8) explica entre el 6 y $13 \%$ de la variación de la inflación diez periodos después del choque. Sin embargo, en dos de estos modelos los efectos no son significativos, que es cuando se utilizan M2 + CDT y la base monetaria como medios de pago (modelos 2 y 4$)$.

Cuando se utiliza la tasa de intervención en diferencias (modelos 1, 3, 5, 7) los efectos de choques de política monetaria explican entre el 10 y el $12 \%$ de la variación de la inflación diez periodos después del choque. En los cuatro modelos estos porcentajes de cambio son significativos.

Por el lado del crecimiento económico, cuando la tasa de intervención está en niveles los choques de política monetaria explican entre el 30 y el $33 \%$ de la variación de éste, diez trimestres después del choque. Cuando la tasa de intervención está en diferencias los choques explican entre el 12 y el $17 \%$ de la variación del crecimiento económico. En los ocho modelos estos efectos son significativos.
Tabla 2. Descomposición de varianza (variable m2 + cdt) ${ }^{24}$.

Bessilts stor saral

\begin{tabular}{|c|c|c|c|c|c|c|}
\hline step & $\begin{array}{l}\text { (1) } \\
\text { sfend }\end{array}$ & $\begin{array}{r}\text { (1) } \\
\text { Lover }\end{array}$ & $\begin{array}{r}\text { (1) } \\
\text { Opper }\end{array}$ & $\begin{array}{l}\text { (2) } \\
\text { sfend }\end{array}$ & $\begin{array}{l}\text { (2) } \\
\text { Lover }\end{array}$ & $\begin{array}{l}\text { (2) } \\
\text { apper }\end{array}$ \\
\hline 0 & 0 & 0 & 0 & 0 & 0 & 0 \\
\hline 1 & $8.20-36$ & 8.5e-36 & $3.2 e-36$ & 0 & 0 & 0 \\
\hline 2 & .026353 & -.022835 & .075553 & .042466 & -.054823 & .139756 \\
\hline 3 & . OSSSAS & $-.004+404$ & .124093 & .108553 & -.007856 & .224375 \\
\hline 4 & .079676 & .010212 & .14914 & .256093 & .021703 & .287484 \\
\hline 5 & .087794 & .02148 & .154107 & .165615 & .127853 & $.3033 \pi$ \\
\hline 6 & .0308377 & .023967 & .15778 & .181267 & .031213 & .331316 \\
\hline 7 & .214203 & .038815 & .183532 & $.17 \% 5$ & . M20234 & .323816 \\
\hline 8 & .272192 & .DHSH & 20973 & .178811 & .208183 & .32938 \\
\hline 3 & .224865 & ه33935 & .209735 & .17825 & .02745 & .32305 \\
\hline 10 & .124455 & .04 & .208303 & .176832 & .266617 & .327137 \\
\hline
\end{tabular}

35s lover and qper bounds reperted

(1) irfnane = srarl, inpolse $=$ dinte, and response $=$ dloipe

(2) irthane $=$ srarl, inpolse $=$ dinte, and response $=$ dlopib

Pesolts srou stare

\begin{tabular}{|c|c|c|c|c|c|c|}
\hline step & $\begin{array}{l}\text { (1) } \\
\text { sfend }\end{array}$ & $\begin{array}{l}\text { (d) } \\
\text { Lorer }\end{array}$ & $\begin{array}{r}\text { (2) } \\
\text { Opper }\end{array}$ & $\begin{array}{l}\text { (2) } \\
\text { shend }\end{array}$ & $\begin{array}{r}\text { (2) } \\
\text { Lorer }\end{array}$ & $\begin{array}{r}\text { (2) } \\
\text { Opper }\end{array}$ \\
\hline 0 & 0 & 0 & 0 & 0 & 0 & 0 \\
\hline 1 & 0 & 0 & 0 & 0 & 0 & 0 \\
\hline 2 & .042373 & -.0167 & .102646 & .0617 & -.054438 & $.17 n 853$ \\
\hline 3 & .043313 & -.011638 & .0395366 & .16885 & .contes & .289316 \\
\hline 4 & .055496 & -.008781 & $.119 m 2$ & .243354 & .065357 & .420751 \\
\hline 5 & .048887 & -.003223 & .106338 & .267001 & .062438 & .472513 \\
\hline 6 & .sms & -.098587 & .1052888 & .291366 & .065331 & .517402 \\
\hline 7 & .058523 &.$- \infty 6181$ & .123428 & .23549 & .057635 & .533264 \\
\hline 8 & . 071376 & -.003328 & .15286 & .293617 & .052034 &. $\sin 4$ \\
\hline 3 & .065538 & -.012397 & .143532 & .30205 & .048646 & .555453 \\
\hline 10 & .067239 & -.009708 & .144305 & .30217 & .045375 & .553066 \\
\hline
\end{tabular}

ISS lover and wper bounds reparted

(1) irchame $=$ srar 2, inpalse $=$ inte, and response $=$ dlaipc

(2) irchume $=$ star2, inpolse $=$ inte, and response $=$ dlopib 


\section{Conclusiones}

En este trabajo se analizaron las relaciones contemporáneas y los efectos dinámicos de política monetaria en Colombia, vía tasa de intervención del Banco de la República.

Como modelo macroeconómico de comportamiento estático se utilizó el AD-AS (demanda agregada-oferta agregada) y se contrastaron las relaciones contemporáneas entre las variables endógenas del modelo.

Para analizar las relaciones contemporáneas y los efectos dinámicos se utilizó modelos econométricos VAR estructural con restricciones de corto plazo, basados en el modelo AD-AS, en los cuales se calculan las matrices de relaciones contemporáneas y los efectos dinámicos de choques de la variación de la tasa de intervención del Banco de la República en las variables inflación y crecimiento económico.

Tanto el análisis contemporáneo como el dinámico partieron de la especificación de ocho modelos, según las variables que se utilicen como medios de pago y según si la tasa de intervención esté en niveles o en diferencias.

Los resultados de la relación contemporánea entre la variable tasa de intervención del banco frente al crecimiento económico, indican que hay una relación positiva pero no significativa entre el crecimiento económico y la tasa de intervención. Aunque el signo es el esperado por la teoría económica no hay una relación significativa entre estas dos variables en términos contemporáneos. Una posible explicación de esta relación contra-intuitiva es que el Banco de la Republica tiene como variable objetivo a la inflación más que al crecimiento económico, por lo tanto es lógico el resultado encontrado.

También se encontró que hay una relación positiva y significativa contemporánea entre la tasa de intervención del Banco de la República y la inflación. En los ocho modelos esta relación es significativa. La explicación de lo anterior es que el Banco de la República tiene en cuenta a la tasa de inflación tanto presente como futura para sus decisiones de política monetaria.
En otros resultados encontrados, en términos contemporáneos, se puede decir que hay una relación positiva pero no significativa entre la inflación y el crecimiento económico en los ocho modelos analizados (curva de oferta agregada), por lo tanto se puede afirmar que por los resultados encontrados los precios son rígidos en el corto plazo. Esto indicaría que para Colombia la curva de oferta agregada de corto plazo es horizontal.

Según los resultados encontrados la inflación no es un fenómeno monetario, por lo menos en el corto plazo, dado que hay una relación negativa contemporánea entre estas dos variables.

En términos contemporáneos hay una relación positiva y significativa entre el crecimiento económico y la variación de la cantidad de dinero. Mientras que no hay una relación significativa entre tasa de intervención y cantidad de dinero, lo cual implica que, en términos contemporáneos, las políticas monetarias no explican a la cantidad de dinero.

En el análisis dinámico se comprueba que un choque positivo de una desviación estándar de la tasa de intervención en diferencias, tienen efectos significativos y cíclicos sobre la inflación entre el segundo y tercer trimestre y luego entre el sexto y octavo. Si la tasa de intervención está en niveles los efectos dinámicos no son significativos.

Los choques positivos de una desviación estándar de política monetaria vía tasa de intervención, sobre el crecimiento real de la economía, indican en todos los casos (todos los modelos), que el crecimiento disminuye entre dos y tres trimestres después del choque. Estas disminuciones son significativas.

Al analizar los efectos dinámicos acumulados vía descomposición de varianza, los resultados encontrados indican que ante un aumento de una desviación estándar en la tasa de intervención del Banco de la República, se dan mayores efectos acumulados sobre el crecimiento económico que sobre la inflación. En todos los modelos un choque positivo de la tasa de intervención genera disminuciones estadísticamente significativas sobre el crecimiento de la economía, mientras que solamente cuando la tasa 
está en diferencias se generan disminuciones significativas sobre la inflación.

Estos últimos resultados son contra-intuitivos, ya que la teoría económica clásica sugiere lo contrario, que choques de política monetaria no tiene efectos sobre las variables reales (crecimiento económico), pero sí sobre las nominales (inflación)

\section{Referencias}

Amisano, G. \& Giannini, C. (1997) Topics in Structural VAR Econometrics. Second edition. Berlin: Springer Verlag.

Bernanke, B. (1986) "Alternative Explanations of the Money-Income Correlation". En: Carnegie Rochester Conference Series on Public Policy, Vol. 25, pp. 49-100.

Blanchard, O.J. (1989) "A Traditional Interpretation of Macroeconomic Fluctuations." American Economic Review, Vol. 79, 1146-1164.

Blanchard, O.J, \& Quah, D. (1989) "The Dynamic Effect of Aggregate Demand and Supply Disturbances." En: American Economic Review, Vol 79, 655-673.

Blanchard, O.J, \& Watson, M. W. (1986) "Are Business Cycles All Alike?". En: R. Gordon (ed.): The American Business Cycle: Continuity and Change, NBER and University of Chicago Press.

Campos, J. S. (2006) "Estimación de la Brecha entre el Pib Potencial y el Observado a través de Modelos VAR estructural para
Colombia". En: Archivos de Economía Departamento Nacional de Planeación No.300

Echavarría, J. J. et al. (2009) "Intervenciones Cambiarias y Política Monetaria en Colombia. Un Análisis de VAR Estructural”. En: Borradores de Economía Banco de la República No.580.

González, A. et al. (2011) "Asimetrías del Empleo y el Producto, una Aproximación de Equilibrio General". En: Borradores de Economía Banco de la República No.663

Lütkepohl, H. (1993) Introduction to Multiple Time series Analysis. Second Edition. Berlin: Springer Verlag

Mihira, T. \& Sugihara, S. (2000) "A Structural VAR Analysis of the Monetary Policy in Japan". En: Economic Research Institute.

Misas, M. \& López, E. (1998) "El Producto Potencial en Colombia: Una Estimación bajo VAR Estructural". En: Borradores Semanales de Economía SGEE Banco de la República No.94.

Sarmiento, J \& Ramírez, A. (2005) "Los Costos de la Desinflación en Colombia Según el Modelo de Buiter-Miller". En: Cuadernos de Economía, vol.XXIV, num.43, diciembre.2005, 129-159.

Shapiro, M. \& Watson, M. (1988) "Sources of Business Cycle Fluctuations". En: NBER Macroeconomic Annual, MIT Press, Vol 3, 111-156.

Sims, C.A. (1980) "Macroeconomics and Reality." En: Econométrica, Vol. 48, pp. 1-49.

Sims, C.A. (1986) "Are Forecasting Models Usable for Policy Analisys?". En: Federal Reserve Bank of Minneapolis Quarterly Review, Winter.

Sims, C.A. (1988) "Bayesian Skepticism on Unit Root Econometrics". En: Journal of Economics Dynamics and Control, Vol. 12 , pp. $463-474$. 


\section{ANEXO 1. MATEMÁTICA PARA EL ANÁLISIS DE ESTÁTICA COMPARATIVA EN (11)}

Resolviendo por Cramer (10) el determinante jacobiano es:

$$
|J|=1+(\text { aPYaRP }+ \text { aRY })>0
$$

Los choques de política monetaria son:

$$
\begin{aligned}
\partial Y / \partial \varepsilon M P & =\frac{\left[\begin{array}{cccc}
0 & 0 & a Y R & 0 \\
0 & 1 & 0 & 0 \\
1 & -a R P & 1 & 0 \\
0 & -a M P & a M R & 1
\end{array}\right]}{|J|}=\frac{-a Y R}{|J|}<0 \\
\partial P / \partial \varepsilon M P & =\frac{\left[\begin{array}{cccc}
1 & 0 & a Y R & 0 \\
-a P Y & 0 & 0 & 0 \\
-a R Y & 1 & 1 & 0 \\
-a M Y & 0 & a M R & 1
\end{array}\right]}{|J|}=\frac{- \text { aYRaPY }}{|J|}<0
\end{aligned}
$$

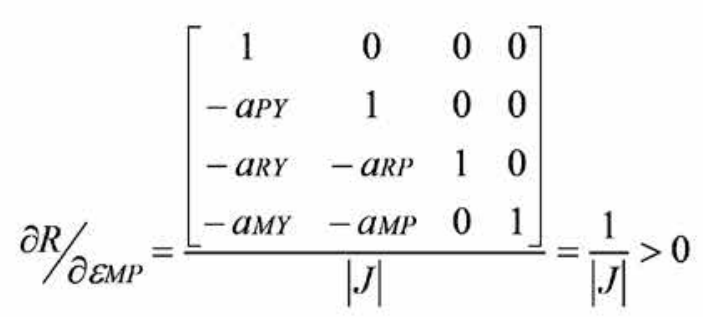

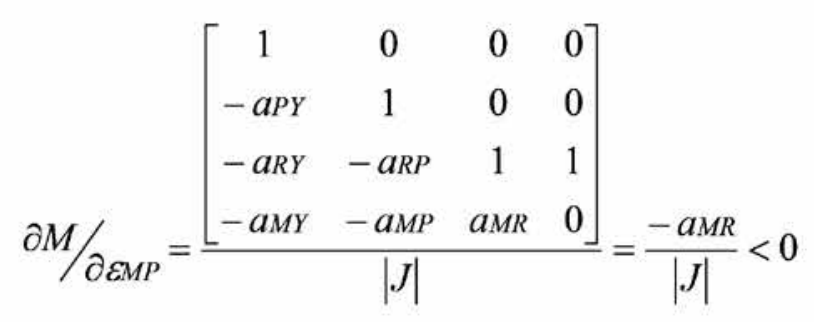




\section{ANEXO 2. RESUMEN DE LAS PRUEBAS DE ESTACIONARIEDAD DE LAS SERIES}

\begin{tabular}{|c|c|c|}
\hline \multicolumn{3}{|c|}{ Pruebas de Raiz Unitaria } \\
\hline & \multicolumn{2}{|c|}{ Estadísticos } \\
\hline Variables & DFA & PP \\
\hline dilogm2c & $-3,09$ & $-7,63$ \\
\hline dilogba & $-3,5$ & $-6,15$ \\
\hline dilogm1 & $-3,77$ & $-6,22$ \\
\hline dilogm2 & $-3,06$ & $-6,77$ \\
\hline dilogpib & $-3,1$ & $-6,96$ \\
\hline dilogipc & $-3,16$ & $-5,22$ \\
\hline inte & $-2,8$ & $-3,31$ \\
\hline diinte & $-4,17$ & $-4,59$ \\
\hline \multicolumn{3}{|c|}{ Valores críticos DFA y PP } \\
\hline $1 \%$ & $5 \%$ & $10 \%$ \\
\hline$-3,534$ & $-2,907$ & $-2,591$ \\
\hline \multicolumn{3}{|c|}{$\begin{array}{l}\text { Nota: resumen de estadísticos calculados con los comandos dfuller y pperron } \\
\text { DFA: Dickey Fuller Aumentado } \\
\text { PP: Phillips Perron } \\
\text { Cálculos de los autores en STATA }\end{array}$} \\
\hline
\end{tabular}

\section{ANEXO 3. RESUMEN DEL NÚMERO DE REZAGOS ÓPTIMO}

\begin{tabular}{|c|c|c|c|}
\hline \multirow{4}{*}{ Modelo } & AIC & HQIC & SBC \\
\cline { 2 - 4 } & \multicolumn{3}{|c|}{ Número de Rezagos } \\
\hline 1 & 8 & 4 & 4 \\
\hline 2 & 8 & 4 & 2 \\
\hline 3 & 8 & 4 & 3 \\
\hline 4 & 8 & 3 & 3 \\
\hline 5 & 8 & 4 & 4 \\
\hline 6 & 5 & 3 & 4 \\
\hline 7 & 8 & 4 & 2 \\
\hline 8 & 8 & 4 & \\
\hline \multicolumn{2}{|c|}{} \\
\hline $\begin{array}{l}\text { Nota: resumen del número de rezagos óptimo de Número de Rezagos } \\
\text { HIIC: Hannan-Quinn Information Criterion } \\
\text { AlC: Akaike Information Criterion } \\
\text { SBC: Schwarts Bayesian Criterion } \\
\text { Cálculos de los autores en STATA }\end{array}$ \\
\hline
\end{tabular}




\title{
ANEXO 4. SALIDA EN STATA DE LA MATRIZ DE COEFICIENTES CONTEMPORÁNEOS DEL MODELO 1
}

\author{
Sanple: $6-65$ \\ kh. of obs $=60$ \\ Overidentified nodel \\ Iog likelihood $=-220.559$
}

\begin{tabular}{|c|c|c|c|c|c|c|}
\hline & Coef. & Std. Rrr. & 2 & $P>|z|$ & [95s Conf. & Interval] \\
\hline /a_1_1 & 1 & (constrained) & & & & \\
\hline $1 a_{-}-1$ & -.082644 & .1290994 & -0.64 & 0.522 & -.3356743 & .1703862 \\
\hline $1 \mathrm{a}_{-}^{3}-1$ & -.1947139 & .1295396 & -1.50 & 0.133 & -.4486068 & .059179 \\
\hline $104-1$ & -.3543836 & .131956 & -2.69 & 0.007 & -.6130127 & -.0957546 \\
\hline $1 \mathrm{a}-12$ & 0 & (constrained) & & & & \\
\hline la_2_ & 1 & (constrained) & & & & \\
\hline $1 / a_{-}^{3}-2$ & -.3316269 & .1290994 & -2.57 & 0.010 & -.5846571 & -.0785966 \\
\hline /a_4 2 & 1.161541 & .1360133 & 8.54 & 0.000 & .8949599 & 1.428122 \\
\hline $1 \mathrm{a}_{-} \mathbf{1}_{3}$ & 0 & (constrained) & & & & \\
\hline $1 a_{2} 23$ & 0 & (constrained) & & & & \\
\hline $1 a_{-} 33$ & 1 & (constrained) & & & & \\
\hline $1 a_{-}^{4}-3$ & .0267584 & .1290994 & 0.21 & 0.836 & -.2262718 & .2797887 \\
\hline /a_1_4 & 0 & (constrained) & & & & \\
\hline /a_2_4 & 0 & (constrained) & & & & \\
\hline $1 a_{-}^{3}-4$ & 0 & (constrained) & & & & \\
\hline $1 a_{-}^{4} 4$ & 1 & (constrained) & & & & \\
\hline /b_1_1 & 1 & (constrained) & & & & \\
\hline /b_2_ & 0 & (constrained) & & & & \\
\hline lb_31 & 0 & (constrained) & & & & \\
\hline $1 b_{-}^{4} 1$ & 0 & (constrained) & & & & \\
\hline$/ b_{-} 12$ & 0 & (constrained) & & & & \\
\hline $1 \mathrm{~b} 22$ & 1 & (constrained) & & & & \\
\hline $1 \mathrm{~b}-32$ & 0 & (constrained) & & & & \\
\hline $1 \mathrm{~b}-42$ & 0 & (constrained) & & & & \\
\hline lb_-13 & 0 & (constrained) & & & & \\
\hline $1 \mathrm{~b} 2-3$ & 0 & (constrained) & & & & \\
\hline$/ b_{-}^{3}-3$ & 1 & (constrained) & & & & \\
\hline$/ b_{-}^{4}-3$ & 0 & (constrained) & & & & \\
\hline /b_14 & 0 & (constrained) & & & & \\
\hline $1 \mathrm{~b}-24$ & 0 & (constrained) & & & & \\
\hline $16-34$ & 0 & (constrained) & & & & \\
\hline /b_4_4 & 1 & (constrained) & & & & \\
\hline
\end{tabular}

LR test of identifying restrictions: $\operatorname{chi} 2(4)=$

1990 Prob $>$ chi2 $=0.000$ 


\section{ANEXO 5. DESCOMPOSICIÓN DE VARIANZA MODELOS 3, 4 Y 5}

\begin{tabular}{|c|c|c|c|c|c|c|}
\hline scep & $\begin{array}{r}\text { (2) } \\
\text { sera }\end{array}$ & $\begin{array}{c}\text { (1) } \\
\text { comer }\end{array}$ & $\begin{array}{r}\text { (2) } \\
\text { vppear }\end{array}$ & $\begin{array}{r}\text { (2) } \\
=\text { zerd }\end{array}$ & $\begin{array}{r}\text { (2) } \\
\text { Lowar }\end{array}$ & $\begin{array}{r}(2) \\
\text { vpper }\end{array}$ \\
\hline 0 & 0 & 0 & 0 & 0 & 0 & 0 \\
\hline 2 & $2.7 e-34$ & $2.6 e-34$ & $2 \cdot 8 e-34$ & 0 & 0 & 0 \\
\hline 2 & .028048 & -.027565 & .08366 & .042346 & -.064583 & .247275 \\
\hline 3 & .043592 & -.026274 & .207353 & .080366 & $-.02 \mathrm{es} 82$ & .289325 \\
\hline 4 & .060972 & -.00652 & .228432 & .233968 & .009872 & .238065 \\
\hline s & .093537 & .02933 & .267784 & .243578 & .00944 & .277726 \\
\hline 6 & .093898 & .020832 & .266964 & .249257 & .006036 & .293678 \\
\hline 7 & .202345 & .026034 & .276656 & .249773 & .004987 & .294559 \\
\hline 8 & .212293 & .030267 & .294328 & .249575 & .004279 & .29487 \\
\hline 9 & .225592 & .032387 & .299796 & .249232 & .003972 & .294489 \\
\hline 20 & .223229 & .03242 & .299047 & .249046 & .004494 & .293598 \\
\hline
\end{tabular}

95. 20wor and upper bounce neportad

(2) Irenane - srax3, 1mpulas - Ainee, and neponse - Alospe

(2) Irmane - spar3, 1mpulse - dince, and moponse - diopib

Reoules frox gvar4

\begin{tabular}{|c|c|c|c|c|c|c|}
\hline step & $\begin{array}{c}\text { (1) } \\
=f e v d\end{array}$ & $\begin{array}{c}\text { (1) } \\
\text { Loxer }\end{array}$ & $\begin{array}{r}\text { (1) } \\
\text { Upper }\end{array}$ & $\begin{array}{r}\text { (2) } \\
\text { fevd }\end{array}$ & $\begin{array}{r}\text { (2) } \\
\text { Loxer }\end{array}$ & $\begin{array}{r}(2) \\
\text { Uppex }\end{array}$ \\
\hline 0 & 0 & 0 & 0 & 0 & 0 & 0 \\
\hline 1 & 0 & 0 & 0 & 0 & 0 & 0 \\
\hline 2 & .048802 & -.024982 & .122587 & .052045 & -.060636 & .166406 \\
\hline 3 & .044854 & -.022676 & .112385 & .164602 & .020682 & .308521 \\
\hline $\begin{array}{l}4 \\
5\end{array}$ & $\begin{array}{l}.058956 \\
.059056\end{array}$ & $\begin{array}{l}-.014699 \\
-.013695\end{array}$ & $\begin{array}{l}.132612 \\
.131806\end{array}$ & $\begin{array}{l}.218819 \\
.266211\end{array}$ & $\begin{array}{l}.033609 \\
.049814\end{array}$ & $\begin{array}{l}.404029 \\
.482608\end{array}$ \\
\hline 6 & .059258 & -.013805 & .132402 & .297807 & .05896 & .536653 \\
\hline 7 & .05727 & -.013975 & .128514 & .313061 & .059463 & .566659 \\
\hline 8 & .071369 & -.009562 & .152299 & .324241 & .060506 & .587975 \\
\hline 9 & .076216 & -.011591 & .164024 & .330107 & .05955 & .600664 \\
\hline 10 & .075456 & -.010269 & .161182 & .332529 & .05798 & .607077 \\
\hline
\end{tabular}

953 lover and upper bounds reported

(1) irfnaxe $=$ svax4, ixpulse $=$ inte, and xesponse $=$ dloipe

(2) irfinase $=$ svar4, ixpulse $=$ inte, and response $=\mathrm{dlopib}$

Resules Irom arass

\begin{tabular}{|c|c|c|c|c|c|c|}
\hline sepp & $\begin{array}{l}\text { (2) } \\
\text { esard }\end{array}$ & $\begin{array}{l}\text { (2) } \\
\text { 2omex }\end{array}$ & $\begin{array}{c}\text { (2) } \\
\text { vpper }\end{array}$ & $\begin{array}{r}\text { (2) } \\
\text { szard }\end{array}$ & $\begin{array}{c}\text { (2) } \\
\text { 2owar }\end{array}$ & $\begin{array}{c}\text { (2) } \\
\text { oppere }\end{array}$ \\
\hline $\begin{array}{l}0 \\
2 \\
2 \\
3 \\
4 \\
5 \\
6 \\
7 \\
8 \\
9 \\
20\end{array}$ & $\begin{array}{l}0 \\
0 \\
.033031 \\
.063364 \\
.072482 \\
.082828 \\
.082498 \\
.203732 \\
.223222 \\
.207649 \\
.207292\end{array}$ & $\begin{array}{l}0 \\
0 \\
-.023901 \\
.000272 \\
.006463 \\
.025829 \\
.026053 \\
.028275 \\
.028224 \\
.023145 \\
.022987\end{array}$ & $\begin{array}{l}0 \\
0 \\
.090002 \\
.226435 \\
.236502 \\
.247826 \\
.248944 \\
.283326 \\
.298028 \\
.292253 \\
.292597\end{array}$ & $\begin{array}{l}0 \\
0 \\
.029074 \\
.06533 \\
.236803 \\
.252025 \\
.266225 \\
.272282 \\
.176984 \\
.182083 \\
.184296\end{array}$ & $\begin{array}{l}0 \\
0 \\
-.036092 \\
-.032806 \\
.024522 \\
.02937 \\
.019032 \\
.029222 \\
.022357 \\
.025274 \\
.027447\end{array}$ & $\begin{array}{l}0 \\
0 \\
.094239 \\
.262465 \\
.259085 \\
.28266 \\
.313397 \\
.323242 \\
.332622 \\
.336992 \\
.340943\end{array}$ \\
\hline
\end{tabular}

St lower and upper bownda reported

(2) Irenase - svars, ispuzse - Asnee, and zesponse - dlospe

(2) Irename - avass. Inpulae - Alnee, and fesponse - diopib 


\section{ANEXO 6. DESCOMPOSICIÓN DE VARIANZA MODELOS 6, 7 Y 8}

Resules from spart

\begin{tabular}{|c|c|c|c|c|c|c|}
\hline step & $\begin{array}{r}\text { (1) } \\
\text { sfevd }\end{array}$ & $\begin{array}{c}\text { (1) } \\
\text { Lover }\end{array}$ & $\begin{array}{r}\text { (1) } \\
\nabla_{\text {pper }}\end{array}$ & $\begin{array}{l}\text { (2) } \\
\text { stevd }\end{array}$ & $\begin{array}{c}\text { (2) } \\
\text { Lover }\end{array}$ & $\begin{array}{r}\text { (2) } \\
\text { Oppex }\end{array}$ \\
\hline 0 & 0 & 0 & 0 & 0 & 0 & 0 \\
\hline 2 & 6.8e-27? & $6.6 e-27$ & $7.1 e-37$ & 0 & 0 & 0 \\
\hline 2 & .074162 & -.007716 & .156041 & .018146 & -.050516 & .086809 \\
\hline 2 & .07158 & -.002032 & .145232 & .112992 & -.005959 & .232944 \\
\hline 4 & .095688 & .009167 & .181909 & .174178 & .008602 & .23967 \\
\hline 5 & .093208 & .007623 & .178982 & .220019 & .017247 & .424291 \\
\hline 6 & .10725 & .012962 & .200537 & .257606 & .028961 & .486251 \\
\hline ? & .109324 & .018161 & .200507 & .274555 & .020401 & .51862 \\
\hline 8 & .122214 & .032101 & .232327 & .288164 & .032872 & .542456 \\
\hline 9 & .131042 & .021511 & .220592 & .297093 & .034691 & .559495 \\
\hline 10 & .127211 & .035719 & .238704 & .200258 & .032831 & .566605 \\
\hline
\end{tabular}

ost lower and upper bounds reported

(1) ixtnase $=$ svare, izpulse = inte, and response $=$ dloipe

(2) irfase $=$ svar6, iapulse $=$ inte, and responge $=$ dlopib

Regules fxoln graz?

\begin{tabular}{|c|c|c|c|c|c|c|}
\hline step & $\begin{array}{l}\text { (1) } \\
\text { sfevd }\end{array}$ & $\begin{array}{c}\text { (1) } \\
\text { Lover }\end{array}$ & $\begin{array}{r}\text { (1) } \\
\sigma_{\text {pper }}\end{array}$ & $\begin{array}{l}\text { (2) } \\
\text { stevd }\end{array}$ & $\begin{array}{l}\text { (2) } \\
\text { Lorex }\end{array}$ & $\begin{array}{r}\text { (2) } \\
\text { Oppex }\end{array}$ \\
\hline 0 & 0 & 0 & 0 & 0 & 0 & 0 \\
\hline 1 & 0 & 0 & 0 & 0 & 0 & 0 \\
\hline 2 & .029612 & -.021782 & .077007 & .0134 & -.044414 & .071215 \\
\hline 3 & .068045 & .005609 & .130481 & .070791 & -.022245 & .163867 \\
\hline 4 & .081238 & .015767 & .146709 & .111285 & .006987 & .215783 \\
\hline 5 & .084925 & .021514 & $.14: 256$ & .120119 & .009228 & .220909 \\
\hline 6 & .006232 & .022452 & .130013 & .129914 & .005514 & .251314 \\
\hline 7 & .110522 & .039596 & .101451 & .120357 & .007899 & .249014 \\
\hline 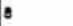 & .122822 & .044255 & .201209 & .12773 & .007084 & .246375 \\
\hline 9 & .117252 & .028981 & .195524 & .127118 & .006425 & .2478 \\
\hline 10 & .116242 & .028904 & .19250 & .125891 & .006002 & .245779 \\
\hline
\end{tabular}

95t lower and upper bounds reported

(1) ixframe $=$ gyar7, implae $=$ dinte, and response $=$ dloipe

(2) irfrase = gvar7, inpulge = dinte, and re sponge $=$ dlopib

Results frox gvare

\begin{tabular}{|c|c|c|c|c|c|c|}
\hline step & $\begin{array}{c}\text { (1) } \\
\text { sfevd }\end{array}$ & $\begin{array}{l}\text { (1) } \\
\text { Lover }\end{array}$ & $\begin{array}{r}\text { (1) } \\
\text { Oppex }\end{array}$ & $\begin{array}{r}\text { (2) } \\
\text { sfevd }\end{array}$ & $\begin{array}{l}\text { (2) } \\
\text { Dorez }\end{array}$ & $\begin{array}{r}(2) \\
\nabla_{\text {ppex }}\end{array}$ \\
\hline 0 & 0 & 0 & 0 & 0 & 0 & 0 \\
\hline 2 & $0.5 e-28$ & $7.9 e-26$ & $9.0 e-36$ & 0 & 0 & 0 \\
\hline 2 & .063629 & -.005939 & .129197 & .011924 & -.04194 & .065789 \\
\hline 2 & .060214 & -.001615 & .122042 & .100222 & -.021221 & .221867 \\
\hline 4 & .062156 & -.002422 & .127745 & .208504 & .042079 & .272929 \\
\hline s & .070211 & .002428 & .126983 & .264150 & .071036 & .458681 \\
\hline 6 & .069332 & .002798 & .124660 & .299145 & .084552 & .512728 \\
\hline 7 & .085675 & $.0114 t 2$ & .159860 & .3046 & .077904 & .531297 \\
\hline is & .098009 & .010929 & .18509 & .206985 & .073296 & .540674 \\
\hline 9 & .090695 & .000572 & .100019 & .210522 & .069374 & $.5516 ?$ \\
\hline 10 & .09948 & .011189 & .187773 & .210261 & .065194 & .555529 \\
\hline
\end{tabular}

ast lover and upper bounds reported

(1) irtrame = syart, izpulge = inte, and responge = dloipe

(2) irfname $=$ svart, iapulge $=$ inte, and responge $=$ dlopib

aeswies cron mare

\begin{tabular}{|c|c|c|c|c|c|c|}
\hline$n=0$ & $\begin{array}{c}\text { (2) } \\
\text { acovd }\end{array}$ & $\begin{array}{l}\text { (2) } \\
\text { torear }\end{array}$ & $\begin{array}{c}\text { (2) } \\
\text { Dpoer }\end{array}$ & $\begin{array}{r}(2) \\
\text { (cord }\end{array}$ & (a) & $\begin{array}{r}\text { (a) } \\
\text { (2) }\end{array}$ \\
\hline$=$ & $\theta$ & o & e & $\theta$ & $\theta$ & $\theta$ \\
\hline 2 & $8.90-30$ & $7.200-30$ & $9.50-30$ & o & 。 & 。 \\
\hline 2 & .063020 & "-00938. & .233287 & .022024 & -.04234 & "0.098 \\
\hline 3 & .060224 & -.002023 & .222042 & .200323 & -.023222 & .222807 \\
\hline 4 & .062280 & -.003433 & .227743 & .208904 & .043070 & (393820, \\
\hline 3 & .070222 & .003438 & .236833 & .264898 & .072038 & .438082 \\
\hline - & .063253 & ".023780 & .234808 & .200249 & .084992 & 4. \\
\hline , & . & .022482 & .299388 & .3860 & .077906 & .932397 \\
\hline$=$ & .008000 & .022020 & .28900 & .356389 & .072290 & .260074 \\
\hline$\therefore$ & .008603 & .008372 & -208020 & .320922 & .009376 & .99267 \\
\hline 20 & .00868 & .021287 & .287773 & .312302 & .009294 & "95293. \\
\hline
\end{tabular}

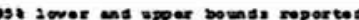

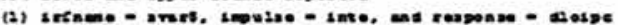

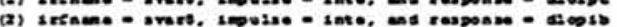

